\title{
Domain walls between gauge theories
}

\author{
S.L. Dubovsky, S.M. Sibiryakov \\ Institute for Nuclear Research of the Russian Academy of Sciences, \\ 60th October Anniversary Prospect, 7a, 117312 Moscow, Russia
}

\begin{abstract}
Noncommutative $U(\mathcal{N})$ gauge theories at different $\mathcal{N}$ may be often thought of as different sectors of a single theory: the $U(1)$ theory possesses a sequence of vacua labeled by an integer parameter $\mathcal{N}$, and the theory in the vicinity of the $\mathcal{N}$-th vacuum coincides with the $U(\mathcal{N})$ noncommutative gauge theory. We construct noncommutative domain walls on fuzzy cylinder, separating vacua with different gauge theories. These domain walls are solutions of BPS equations in gauge theory with an extra term stabilizing the radius of the cylinder. We study properties of the domain walls using adjoint scalar and fundamental fermion fields as probes. We show that the regions on different sides of the wall are not disjoint even in the low energy regime - there are modes penetrating from one region to the other. We find that the wall supports a chiral fermion zero mode. Also, we study non-BPS solution representing a wall and an antiwall, and show that this solution is unstable. We suggest that the domain walls emerge as solutions of matrix model in large class of pp-wave backgrounds with inhomogeneous field strength. In the M-theory language, the domain walls have an interpretation of a stack of branes of fingerstall shape inserted into a stack of cylindrical branes.
\end{abstract}

\section{Introduction}

Recently, field theories on noncommutative (NC) spaces attracted considerable interest (see, e.g., Refs. [1, 2, 3, 4] for recent reviews and references to earlier works). One of the reasons for this interest is the fact that NC gauge theories are non-local and that the group of gauge transformations in $\mathrm{NC}$ theories contains some of the diffeomorphisms ${ }^{1}$. Both non-locality and invariance under diffeomorphisms are expected to hold in the theory of quantum gravity, so one may hope to gain some insight into techniques appropriate in quantum gravity, using $\mathrm{NC}$ theories as a toy model (recall that NC theories are simpler in the sense that non-locality is present there already at the classical level). This hope is further supported by the fact that $\mathrm{NC}$ gauge theories emerge as effective descriptions of string theory in a certain limit [5], and are inherent in the matrix approach to M-theory.

\footnotetext{
${ }^{1}$ Strictly speaking, the latter property holds only for fields in the adjoint representation of gauge group.
} 
Among the consequences of non-locality and invariance under coordinate transformations is the background dependence of both the matter content and space-time interpretation of $\mathrm{NC}$ gauge theory. One illustration of this background dependence is that in many cases $\mathrm{NC}$ gauge theories with different gauge groups $U(\mathcal{N})$ emerge as different sectors of a single theory $[6,7,8,9]$. For instance, it was pointed out in Ref. [6] that $U(1)$ gauge theory on the $\mathrm{NC}$ plane possesses a sequence of vacua labeled by a natural number $\mathcal{N}$ with the following peculiar properties:

i) Every vacuum with $\mathcal{N}>1$ is a highly non-local field configuration from the point of view of the trivial $(\mathcal{N}=1)$ vacuum;

ii) Perturbation theory in the vicinity of the $\mathcal{N}$-th vacuum is equivalent to perturbation theory of the $U(\mathcal{N}) \mathrm{NC}$ gauge theory above its trivial vacuum;

iii) The fact that there are different gauge theories in different vacua cannot be understood as Higgs mechanism. Namely, the action in the vicinity of the $\mathcal{N}$-th vacuum contains $U(\mathcal{N})$ gauge fields only, with no extra massive vector bosons or Higgs fields.

The precise physical meaning of this phenomenon is not clear yet. In the case of $\mathrm{NC}$ plane, it was argued [6] that $\mathcal{N}$ is a superselection parameter, implying that different sectors are completely disconnected from each other.

One way to understand the physical meaning of the parameter $\mathcal{N}$ is to study whether there exist domain walls separating vacua with different values of $\mathcal{N}$. These walls, if any, are expected to exhibit interesting physical properties, since in the low-energy (commutative) limit they would serve as boundaries between regions with different gauge theories inside. On the other hand, the absence of the domain walls would imply that sectors with different $\mathcal{N}$ are disconnected, and $\mathcal{N}$ is indeed just a superselection parameter.

In this paper we study the problem of existence of the domain walls in the context of gauge theory on a fuzzy cylinder introduced in Ref. [8] (see also Ref. [10]). We choose cylindrical geometry because in this case a domain wall normal to the axis of the cylinder has finite energy. Fuzzy cylinder can be thought of as a lattice version of the continuous $\mathrm{NC}$ cylinder studied in Ref. [9]. It is a well-defined NC space which reduces to ordinary cylinder in the commutative limit. An advantadge of the fuzzy cylinder setup compared to the continuous $\mathrm{NC}$ cylinder is that the intriguing phenomenon described above (existence of the sequence of vacua corresponding to different gauge theories) is more transparent in the former case. We elaborate on the relation between the fuzzy and NC cylinders in section 2 .

We find that though configurations of finite energy interpolating between different gauge vacua exist already in pure Yang-Mills theory on the fuzzy cylinder (these configurations 
were first constructed in Ref. [11] and were interpreted there as D-brane junctions), they are unstable and tend to dissolve classically ${ }^{2}$. However, once a gauge-invariant term, stabilizing the radius of the cylinder, is added to the action, the domain walls become stable solutions. They saturate the BPS bound emerging due to the existence of a non-trivial topological charge. We construct explicitly a family of solutions of the BPS equations, that describe domain walls between $U\left(\mathcal{N}_{1}\right)$ and $U\left(\mathcal{N}_{2}\right)$ vacua with arbitrary $\mathcal{N}_{1}$ and $\mathcal{N}_{2}$.

We suggest also a matrix model interpretation of these domain walls. Namely, we argue that a term needed to stabilize the domain wall is generated in a large class of curved gravitational pp-wave backgrounds with inhomogeneous three-form field strength. In the M-theory language, domain wall solutions have an interpretation of a stack of branes of fingerstall shape, inserted into a stack of cylindrical branes.

An interesting property of the domain walls we construct is that the regions with different gauge theories they separate are not disjoint even in the low energy (long wavelength) regime. We illustrate this point by studying the properties of adjoint scalar and fundamental fermion fields in the background of the simplest domain wall between $U(1)$ and $U(2)$ gauge vacua. We show that there are long wavelength modes of these fields, which penetrate from the region with $U(2)$ gauge theory to that with $U(1)$ gauge theory. It is worth stressing that these modes do not belong to the diagonal part of the $U(2)$ group. Other modes, on the contrary, experience total reflection from the wall. In addition, we find that the wall localizes a zero fermionic mode, whose profile can be used as a probe of the shape of the wall.

Finally, we study a wall-antiwall system which separates the cylinder into three regions with $U(1), U(2)$ and again $U(1)$ gauge theories. An unusual property of this system (which is not BPS) is the absence of attraction between the wall and antiwall in the sense that there is a one-parameter family of solutions with equal energies and different distances between the wall and antiwall. However, a tachyonic mode is present in the spectrum of excitations of the wall-antiwall system. The absolute value of its mass squared is exponentially small when the distance between the walls is large. Thus, the wall-antiwall system is almost stable, when the walls are well separated.

This paper is organized as follows. In section 2 we introduce the algebra of functions on the fuzzy cylinder and describe the gauge theory. In section 3 we discuss the topology of domain wall configuration, derive the BPS bound for its energy and solve the corresponding BPS equations. In section 4 we consider scalar and fermion fields in the domain wall background. Wall-antiwall system is studied in section 5. In section 6 we discuss the rela-

\footnotetext{
${ }^{2}$ In Ref. [11] it was suggested that these configurations may be stabilized by quantum effects.
} 
tion between our model and matrix theory in curved background. The concluding section 7 contains brief summary and discussion of our results.

\section{Fuzzy cylinder}

\subsection{Algebra of functions on the fuzzy cylinder}

To introduce algebra $\mathcal{A}_{C}$ of functions on the NC cylinder and algebra $\mathcal{A}_{F}$ of functions on the fuzzy cylinder, it is convenient to start with the "master" algebra $\mathcal{A}$ generated by three elements $\mathbf{x}, \mathbf{y}, \mathbf{z}$, obeying the following commutation relations

$$
[\mathbf{z}, \mathbf{x}]=i l \mathbf{y}, \quad[\mathbf{z}, \mathbf{y}]=-i l \mathbf{x}, \quad[\mathbf{x}, \mathbf{y}]=0
$$

where the parameter $l$ is the scale of noncommutativity and operators $\mathbf{x}, \mathbf{y}$ and $\mathbf{z}$ may be thought of as the coordinates of a three-dimensional NC space where NC and fuzzy cylinders are embedded into. It is convenient to introduce linear combinations

$$
\mathbf{x}_{+}=\mathbf{x}+i \mathbf{y}, \quad \mathbf{x}_{-}=\mathbf{x}-i \mathbf{y}
$$

Commutation relations for these elements have the form

$$
\left[\mathbf{z}, \mathbf{x}_{+}\right]=l \mathbf{x}_{+}, \quad\left[\mathbf{z}, \mathbf{x}_{-}\right]=-l \mathbf{x}_{-}, \quad\left[\mathbf{x}_{+}, \mathbf{x}_{-}\right]=0
$$

Irreducible representations of the algebra $\mathcal{A}$ are labeled by the eigenvalues of the two central elements

$$
T_{1}=\mathbf{x}_{+} \mathbf{x}_{-}=\mathbf{x}^{2}+\mathbf{y}^{2}, \quad T_{2}=\mathrm{e}^{2 \pi i \mathbf{z} / l}
$$

The eigenvalue of the first central element $T_{1}$ is natural to interprete as radius squared of a cylinder. Thus, to introduce algebra $\mathcal{A}_{C}$ of functions on the $\mathrm{NC}$ cylinder of radius $\rho$, one sets

$$
T_{1}=\rho^{2}
$$

More formally, algebra $\mathcal{A}_{C}$ can be defined as a factor-algebra

$$
\mathcal{A}_{C}=\mathcal{A} /\left\{T_{1}-\rho^{2}\right\}
$$

where $\left\{T_{1}-\rho^{2}\right\}$ is a subalgebra of algebra $\mathcal{A}$ generated by the element $\left(T_{1}-\rho^{2}\right)$. For recent study of gauge theory on the NC cylinder see Ref. [9]. 
On the other hand, by fixing the value of the second central element

$$
T_{2}=\mathrm{e}^{2 \pi i z_{0} / l}
$$

one obtains a collection of planes parallel to $(x, y)$-plane with $z$-coordinate equal to $\left(z_{0}+n l\right)$, $n \in \mathbb{Z}$. Algebra $\mathcal{A}_{F}$ of the fuzzy cylinder (see, e.g., Refs. [8, 10]) is obtained by fixing the values of both central elements $T_{1}$ and $T_{2}$. In other words, one defines algebra $\mathcal{A}_{F}$ as the following factor-algebra,

$$
\mathcal{A}_{F}=\mathcal{A}_{\mathcal{C}} /\left\{T_{2}-1\right\}=\mathcal{A} /\left\{T_{1}-\rho^{2}, T_{2}-1\right\}
$$

where without loss of generality we set $z_{0}=0$. This algebra may be realized as the algebra of operators acting in a Hilbert space $H$ with basis vectors $|n\rangle, n \in \mathbb{Z}$,

$$
\mathbf{z}=l \sum_{n=-\infty}^{\infty} n|n\rangle\left\langle n\left|, \quad \mathbf{x}_{+}=\rho \sum_{n=-\infty}^{\infty}\right| n+1\right\rangle\left\langle n\left|, \quad \mathbf{x}_{-}=\rho \sum_{n=-\infty}^{\infty}\right| n-1\right\rangle\langle n| .
$$

It is clear from the above discussion that fuzzy cylinder can be thought of as a cylindrical semi-lattice with continuos coordinate $\theta$ defined by

$$
x_{ \pm}=\rho \mathrm{e}^{ \pm i \theta}
$$

and discrete coordinate $z$ with spacing $l$.

To make this picture more transparent it is instructive to introduce symbols of the operators in the Hilbert space $H$. We will use the symmetric ordering which maps functional exponents into operator exponents

$$
\mathrm{e}^{i(k z+N \theta)} \rightarrow \mathrm{e}^{i(k \mathbf{z}+N \boldsymbol{\theta})}=\mathrm{e}^{-i k N l / 2} \mathrm{e}^{i k \mathbf{z}}\left(\frac{\mathbf{x}_{+}}{\rho}\right)^{N}
$$

Then it is straightforward to obtain the following relation between an arbitrary operator $f=\sum f_{n m}|n\rangle\langle m|$ and its symbol $\tilde{f}(z, \theta)$

$$
\tilde{f}(z, \theta)=l \sum_{n, m=-\infty}^{\infty} \int_{-\frac{\pi}{l}}^{\frac{\pi}{l}} \frac{d k}{2 \pi} f_{n m} \mathrm{e}^{i k\left(z-\frac{l(n+m)}{2}\right)+i(n-m) \theta}
$$

Note that the integration over $k$ has finite range $k \in(-\pi / l, \pi / l)$. Consequently, the symbol $\tilde{f}(z, \theta)$ is uniquely determined by its values on the cylindrical semi-lattice with lattice points

$$
z=n l, \quad n \in \mathbb{Z} .
$$


Equivalently, one may view the map (2) as a correspondence between operators in $H$ and functions on the cylinder, whose Fourier components along $z$-coordinate are cut off at the scale $\pi / l$. Clearly, a symbol $\tilde{f}(z, \theta)$ considered as a function of the continuous variable $z$ contains the same amount of information as its values on the lattice $\tilde{f}(\ln , \theta)$, but many formulae simplify when written in terms of functions of the continuous variable.

The map (2) implies the following relation between trace of the operator and integral of the symbol,

$$
2 \pi \rho l \operatorname{Tr} f=\int \rho d \theta d z \tilde{f}=\rho l \sum_{n} \int d \theta \tilde{f}(\ln , \theta) .
$$

Let us now define derivatives of functions on the fuzzy cylinder. It is straightforward to check that differentiation of symbols with respect to $\theta$-coordinate translates into the following inner derivation in the operator language,

$$
\partial_{3} \tilde{f} \equiv \frac{i}{l} \widetilde{[\mathbf{z}, f]}=\frac{\partial \tilde{f}}{\partial \theta} .
$$

This relation has the same form as in the case of $\mathrm{NC}$ cylinder (see, e.g. Ref. [9]). However, it is impossible to define a derivative along $z$-direction. Indeed, in the language of symbols we consider functions on the lattice in $z$ direction, so it is natural to expect that some discretized version of the derivative in $z$ direction emerges. In the operator language, a naive attempt to define $z$-derivative would contradict the Leibnitz rule because of the constraint $T_{2}=1$.

On the other hand, from the algebraic point of view it is natural to consider on equal footing the following three inner derivations of the algebra $\mathcal{A}_{F}$

$$
\partial_{1} f=\frac{i}{l}[\mathbf{x}, f], \quad \partial_{2} f=-\frac{i}{l}[\mathbf{y}, f], \quad \partial_{3} f=\frac{i}{l}[\mathbf{z}, f] .
$$

As pointed out above, the last derivation $\partial_{3}$ corresponds to differentiation with respect to $\theta$ in terms of symbols. Furthemore, it is straightforward to check that

$$
\begin{gathered}
\partial_{1} \tilde{f} \equiv \frac{i}{l[\widetilde{\mathbf{x}, f]}}=y d_{z} \tilde{f} \\
\partial_{2} \tilde{f} \equiv-\frac{i}{l} \widetilde{[\mathbf{y}, f]}=x d_{z} \tilde{f},
\end{gathered}
$$

where finite-difference derivative $d_{z}$ is defined as

$$
d_{z} \tilde{f}=\frac{\tilde{f}(z+l / 2)-\tilde{f}(z-l / 2)}{l} .
$$

Note that this derivative has a simple form when written in terms of symbol $\tilde{f}(z)$ considered as a function of continuous variable $z$. However, written as a lattice derivative this operator 
has the following highly non-local form

$$
d_{z} \tilde{f}(n l)=-\frac{1}{\pi l} \sum_{k}(-1)^{k} k \frac{\tilde{f}((n+k) l)}{k^{2}-1 / 4} .
$$

This property illustrates the fact that the formulation in terms of functions $\tilde{f}(z)$ of continuous variable is often more convenient than the lattice formulation.

\subsection{Scalar field on the fuzzy cylinder}

To get accustomed to physics of fuzzy cylinder, let us consider a free scalar field theory on it. The action has the following form

$$
S=\int d t 2 \pi \rho l \operatorname{Tr} \frac{1}{2}\left(\left(\partial_{0} \phi\right)^{2}-\frac{1}{\rho^{2}}\left(\left(\partial_{1} \phi\right)^{2}+\left(\partial_{2} \phi\right)^{2}+\left(\partial_{3} \phi\right)^{2}\right)-m^{2} \phi^{2}\right) .
$$

The spectrum of this theory can be determined in two different ways. First, one can rewrite the action (6) in terms of the symbol of the operator $\phi$. Using Eqs. (3) - (5) one has

$$
S=\int d t \rho d \theta d z \frac{1}{2}\left(\left(\partial_{0} \tilde{\phi}\right)^{2}-\frac{1}{\rho^{2}}\left(\partial_{\theta} \tilde{\phi}\right)^{2}-\left(d_{z} \tilde{\phi}\right)^{2}-m^{2} \tilde{\phi}^{2}\right) .
$$

Solutions of the field equations following from the action (7) have the form of waves

$$
\tilde{\phi} \propto \mathrm{e}^{-i \omega t+i k z+i N \theta}, \quad k \in\left(-\frac{\pi}{l}, \frac{\pi}{l}\right)
$$

with the dispersion relation

$$
\omega^{2}=\frac{N^{2}}{\rho^{2}}+\left(\frac{2}{l} \sin \frac{k l}{2}\right)^{2}+m^{2} .
$$

In the long wavelength limit $k \ll 1 / l$ we recover the usual dispersion relation for scalar waves on the cylinder.

It is instructive to obtain the dispersion relation (8) in the operator approach. Variation of the action (6) yields the following field equation

$$
-\partial_{0}^{2} \phi=\frac{1}{\rho^{2} l^{2}}\left([\mathbf{z},[\mathbf{z}, \phi]]+\frac{1}{2}\left[\mathbf{x}_{+},\left[\mathbf{x}_{-}, \phi\right]\right]+\frac{1}{2}\left[\mathbf{x}_{-},\left[\mathbf{x}_{+}, \phi\right]\right]\right)+m^{2} \phi^{2} .
$$

In components $\phi_{n m}$ of the operator $\phi=\sum \phi_{n m}|n\rangle\langle m|$, this equation takes the form

$$
-\partial_{0}^{2} \phi_{n m}=\frac{1}{\rho^{2}}(n-m)^{2} \phi_{n m}+\frac{1}{l^{2}}\left(2 \phi_{n m}-\phi_{n+1, m+1}-\phi_{n-1, m-1}\right)+m^{2} \phi_{n m} .
$$


The system of equations (9) decomposes into independent recursion equations along diagonals of the matrix $\phi$. This corresponds to the Kaluza-Klein decomposition over the compact variable $\theta$. Let us consider equation (9) along the $N$ th diagonal, $n-m=N$, describing the $N$ th KK-mode,

$$
-\partial_{0}^{2} \phi_{n}=\frac{1}{\rho^{2}} N^{2} \phi_{n}+\frac{1}{l^{2}}\left(2 \phi_{n}-\phi_{n+1}-\phi_{n-1}\right)+m^{2} \phi_{n}
$$

where we have set $\phi_{n} \equiv \phi_{n, n+N}$. We immediately find that the Ansatz $\phi_{n}=\phi_{0} \mathrm{e}^{-i \omega t+i k l n}$ is consistent with this equation, yielding the dispersion relation (8).

\subsection{Gauge theory on the fuzzy cylinder}

Let us now describe $U(\mathcal{N})$ gauge theory on the fuzzy cylinder. To this end we consider a field $\psi$ transforming under the fundamental representation of $U(\mathcal{N})$. This field belongs to the direct sum of $\mathcal{N}$ copies of the algebra $\mathcal{A}_{F}$

$$
\psi \in \mathbb{C}^{\mathcal{N}} \otimes \mathcal{A}_{F} \equiv \bigoplus_{i=1}^{\mathcal{N}} \mathcal{A}_{F} .
$$

Gauge transformations are defined as follows,

$$
\psi \rightarrow U \psi
$$

where $U$ is a unitary operator acting in $\mathbb{C}^{\mathcal{N}} \otimes \mathcal{A}_{F}$ (equipped with scalar product $\left(\psi_{1}, \psi_{2}\right)=$ $\left.\operatorname{Tr}\left(\psi_{1}^{+} \psi_{2}\right)\right)$. Covariant connection is introduced in the following way

$$
\begin{aligned}
\nabla\left(\partial_{1}\right) \psi & =\frac{i}{l}(\mathbf{X} \psi-\psi \mathbf{x}) \\
\nabla\left(\partial_{2}\right) \psi & =-\frac{i}{l}(\mathbf{Y} \psi-\psi \mathbf{y}) \\
\nabla\left(\partial_{3}\right) \psi & =\frac{i}{l}(\mathbf{Z} \psi-\psi \mathbf{z}),
\end{aligned}
$$

where covariant coordinates $\mathbf{X}, \mathbf{Y}, \mathbf{Z}$ are Hermitian $\mathcal{N} \times \mathcal{N}$ matrices with entries in $\mathcal{A}_{F}$. They transform under the adjoint representation of the gauge group, $\mathbf{X} \rightarrow U \mathbf{X} U^{+}$, etc. The covariant strength tensor is defined in the usual way, ${ }^{3}$

$$
F_{i j}=\left[\nabla\left(\partial_{i}\right), \nabla\left(\partial_{j}\right)\right]-\nabla\left(\left[\partial_{i}, \partial_{j}\right]\right) .
$$

\footnotetext{
${ }^{3}$ The second term in the r.h.s. of Eq. (10) may appear somewhat unusual. In fact, it is present in the conventional field theory as well. In commutative field theories this term ensures that $F_{i j}$ is a tensor function (not a differential operator) when vector fields $\partial_{i}$ and $\partial_{j}$ do not commute.
} 
Thus, we obtain

$$
\begin{gathered}
F_{12}=\frac{1}{l^{2}}[\mathbf{X}, \mathbf{Y}] \\
F_{13}=-\frac{1}{l^{2}}([\mathbf{X}, \mathbf{Z}]+i l \mathbf{Y}) \\
F_{23}=\frac{1}{l^{2}}([\mathbf{Y}, \mathbf{Z}]-i l \mathbf{X}) \\
F_{0 j}=\frac{i}{l}\left(\partial_{0} \mathbf{X}_{j}-i\left[A_{0}, \mathbf{X}_{j}\right]\right), \quad \mathbf{X}_{j}=\mathbf{X}, \mathbf{Y}, \mathbf{Z} .
\end{gathered}
$$

Now, it is straightforward to write down the Yang-Mills action,

$$
S=\frac{2 \pi \rho l}{g^{2}} \operatorname{Tr}\left(-\frac{1}{\rho^{2}} F_{0 i}^{2}+\frac{1}{2 \rho^{4}} F_{i j}^{2}\right),
$$

where summation is assumed over indices $i, j=1,2,3$. To figure out the commutative limit of the theory, it is convenient to decompose the covariant coordinates in the following way,

$$
\begin{aligned}
\mathbf{X} & =\mathbf{x}-\frac{l}{2}\left(\mathbf{y} A_{z}+A_{z} \mathbf{y}\right)+\frac{l}{2}\left(\mathbf{x} A_{\rho}+A_{\rho} \mathbf{x}\right) \\
\mathbf{Y} & =\mathbf{y}+\frac{l}{2}\left(\mathbf{x} A_{z}+A_{z} \mathbf{x}\right)+\frac{l}{2}\left(\mathbf{y} A_{\rho}+A_{\rho} \mathbf{y}\right) \\
\mathbf{Z} & =\mathbf{z}-l A_{\theta} .
\end{aligned}
$$

Substituting expressions (13) into Eqs. (11) and taking the limit $l \rightarrow 0$, one obtains

$$
\begin{gathered}
F_{12}=-i \rho^{2} D_{z} A_{\rho} \\
F_{13}=i \rho\left(\sin \theta F_{\theta z}-\cos \theta D_{\theta} A_{\rho}\right) \\
F_{23}=i \rho\left(\cos \theta F_{\theta z}+\sin \theta D_{\theta} A_{\rho}\right) \\
F_{01}=i \rho\left(-\sin \theta F_{t z}+\cos \theta D_{t} A_{\rho}\right) \\
F_{02}=i \rho\left(\cos \theta F_{t z}+\sin \theta D_{t} A_{\rho}\right) \\
F_{03}=-i F_{t \theta},
\end{gathered}
$$

where

$$
F_{\alpha \beta}=\partial_{\alpha} A_{\beta}-\partial_{\beta} A_{\alpha}-i\left[A_{\alpha}, A_{\beta}\right], \quad D_{\alpha} A_{\rho}=\partial_{\alpha} A_{\rho}-i\left[A_{\alpha}, A_{\rho}\right], \quad \alpha, \beta=t, \theta, z .
$$

Thus, in the commutative limit one obtains the usual Yang-Mills theory on the cylinder, coupled to an adjoint scalar field $A_{\rho}$. The presence of such a scalar is a consequence of the fact that the algebra of the fuzzy cylinder $\mathcal{A}_{F}$ possesses three independent derivations instead of two, and hence there are three gauge fields in the NC gauge theory. 
Now, let us note the following peculiar property of NC gauge theory on the fuzzy cylin$\operatorname{der}^{4}$. Consider $U(1)$ gauge theory. Then for any natural $\mathcal{N}$, there exists a vacuum in this theory, such that the theory above this vacuum is identical to $U(\mathcal{N})$ gauge theory above its trivial vacuum. Namely, the vacuum corresponding to $U(\mathcal{N})$ theory has the form

$$
\begin{aligned}
& \mathbf{Z}=l \sum_{n=-\infty}^{\infty} n \sum_{a=1}^{\mathcal{N}}|n \mathcal{N}+a\rangle\langle n \mathcal{N}+a| \\
& \mathbf{X}_{+}=\rho \sum_{n=-\infty}^{\infty} \sum_{a=1}^{\mathcal{N}}|(n+1) \mathcal{N}+a\rangle\langle n \mathcal{N}+a| \\
& A_{0}=0 .
\end{aligned}
$$

It is straightforward to check, that the energy of configuration (14) is equal to zero, and thus it indeed describes a vacuum in the $U(1)$ theory. Equivalence between this vacuum and the trivial vacuum in $U(\mathcal{N})$ theory can be established using an isomorphism $S: \mathbb{C}^{\mathcal{N}} \otimes H \rightarrow H$, defined on the basis vectors as

$$
|a\rangle \otimes|n\rangle \stackrel{S}{\mapsto}|n \mathcal{N}+a\rangle
$$

which maps operators (14a), (14b) into the following operators acting in a direct sum of $\mathcal{N}$ Hilbert spaces

$$
\begin{gathered}
\mathbf{Z}=l\left(\sum_{n=-\infty}^{\infty} n|n\rangle\langle n|\right) \cdot \mathbf{1} \\
\mathbf{X}_{+}=\rho\left(\sum_{n=-\infty}^{\infty}|(n+1)\rangle\langle n|\right) \cdot \mathbf{1},
\end{gathered}
$$

where 1 stands for the unit $\mathcal{N} \times \mathcal{N}$ matrix. Field configuration given by Eqs. (15) describes a trivial vacuum in the $U(\mathcal{N})$ theory. Using the same trick for the field configurations describing fluctuations above the vacuum given by Eqs. (14), one observes that the action governing these fluctuations is equivalent to the action describing fluctuations of the $U(\mathcal{N})$ theory in the vicinity of the trivial vacuum.

Thus, the $U(1)$ gauge theory on the fuzzy cylinder has an infinite set of vacua labeled by $\mathcal{N}=1,2, \ldots$ It is natural to wonder whether these vacua correspond to different superselection sectors, or are different phases of one and the same theory. In particular, one may ask

\footnotetext{
${ }^{4}$ This property is generic for gauge theories on a non-compact NC manifold. In the case of NC plane it was discussed in Ref. [6], for NC cylinder in Ref. [9]; for earlier discussions of this property for fuzzy cylinder see Refs. $[8,11]$.
} 
whether there exist solutions (domain walls) interpolating between different vacua. In the next section we answer affirmatively to this question and explicitly find such domain wall solutions.

\section{Domain wall}

In what follows it is convenient to use the dimensionless variables

$$
Z=\frac{1}{l} \mathbf{Z}, \quad X_{ \pm}=\frac{1}{\rho} \mathbf{X}_{ \pm}
$$

We search for a static solution of $U(1)$ gauge theory on the fuzzy cylinder in the form of a domain wall, separating vacua corresponding to different gauge theories. We will work in the gauge $A_{0}=0$. For concreteness we first concentrate on the case of a domain wall between $U(1)$ gauge theory in the region $z<0$ and $U(2)$ gauge theory in the region $z>0$. Then the asymptotics of the domain wall are given by Eqs. (1) and (14), respectively,

$$
\begin{gathered}
Z=\sum n|n\rangle\left\langle n\left|, \quad X_{+}=\sum\right| n+1\right\rangle\langle n|, \quad n \rightarrow-\infty \\
Z=\sum n(|2 n-1\rangle\langle 2 n-1|+| 2 n\rangle\langle 2 n|), \quad X_{+}=\sum|n+2\rangle\langle n|, \quad n \rightarrow+\infty .
\end{gathered}
$$

Let us note that field configurations of finite energy, possessing asymptotics (17) do exist [11]. A simple example is

$$
\begin{aligned}
& Z=\sum_{n=-\infty}^{0} n|n\rangle\langle n|+\sum_{n=1}^{\infty} n(|2 n-1\rangle\langle 2 n-1|+| 2 n\rangle\langle 2 n|) \\
& X_{+}=\sum_{n=-\infty}^{0}|n+1\rangle\left\langle n\left|+\sum_{n=1}^{\infty}\right| n+2\right\rangle\langle n|
\end{aligned}
$$

This configuration is not a static solution of the field equations for gauge theory on the fuzzy cylinder with action given by Eq. (12). Nevertheless, it demonstrates non-trivial topological properties one may expect for the domain wall solution. In NC field theory the role of topological invariants is played by traces of commutators. Indeed, the latter do not change under small variations of operators they depend on. For configuration (18) we have

$$
\operatorname{Tr}\left[X_{+}, X_{-}\right]=-1
$$

The existence of such topological charge suggests a strategy of the search for the domain wall solution: one may try to obtain a BPS bound for the energy functional following from 
Eq. (12) in the topological sector determined by Eq. (19). However, as we will see below, the action (12) as it stands does not admit BPS solutions with desired properties — the domain wall tends to dissolve.

To stabilize the domain wall, let us introduce the following additional term into the gauge theory action

$$
S_{m}=\int d t \frac{2 \pi \rho l}{g^{2}} \operatorname{Tr}\left(-\frac{m^{2}}{16 l^{2}}\left(X_{+} X_{-}+X_{-} X_{+}-2\right)^{2}\right) .
$$

This term has simple physical effect: it stabilizes the radius of the cylinder. In the commutative limit it becomes a mass term for the adjoint scalar field $A_{\rho}$,

$$
S_{m} \rightarrow \int d t \rho d \theta d z\left(-\frac{m^{2}}{g^{2}} A_{\rho}^{2}\right) \quad \text { as } l \rightarrow 0
$$

With this term added, the static energy takes the following form,

$$
\mathcal{E}=\frac{2 \pi}{g^{2} \rho l} \operatorname{Tr}\left(\left|\left[Z, X_{+}\right]-X_{+}\right|^{2}+\frac{\lambda^{2}}{4}\left[X_{+}, X_{-}\right]^{2}+\frac{\mu^{2}}{4}\left(X_{+} X_{-}+X_{-} X_{+}-2\right)^{2}\right)
$$

where we introduced dimensionless parameters

$$
\lambda=\frac{\rho}{l}, \quad \mu=\frac{m \rho}{2} .
$$

This expression can be rewritten in the BPS form

$$
\begin{aligned}
\mathcal{E}=\frac{2 \pi}{g^{2} \rho l} \operatorname{Tr}\left(\left|\left[Z, X_{+}\right]-X_{+}\right|^{2}+\right. & \left(\frac{\lambda}{2}\left[X_{+}, X_{-}\right] \pm \frac{\mu}{2}\left(\left\{X_{+}, X_{-}\right\}-2\right)\right)^{2} \\
& \left.\mp \frac{\lambda \mu}{4}\left\{\left[X_{+}, X_{-}\right],\left(\left\{X_{+}, X_{-}\right\}-2\right)\right\}\right) \geq \mp \frac{2 \pi}{g^{2} \rho l} \frac{\lambda \mu}{2} Q
\end{aligned}
$$

where braces stand for anti-commutators, and

$$
Q=\frac{1}{2} \operatorname{Tr}\left\{\left[X_{+}, X_{-}\right],\left(\left\{X_{+}, X_{-}\right\}-2\right)\right\}=\operatorname{Tr}\left[X_{+},\left(X_{-} X_{+} X_{-}-2 X_{-}\right)\right]
$$

is a topological charge. For the configuration (18) the topological charge is equal to one. Energy in each topological sector is minimized by the solution of BPS equations, which follow from Eq. (23),

$$
\begin{aligned}
& {\left[Z, X_{+}\right]-X_{+}=0} \\
& {\left[Z, X_{-}\right]+X_{-}=0} \\
& (\lambda+\mu) X_{-} X_{+} \pm(\lambda-\mu) X_{+} X_{-}=2 \mu
\end{aligned}
$$


These equations can be considered as defining an algebra with three generators $Z, X_{+}, X_{-}$. Each solution of Eqs. (25) decomposes into direct sum of operators acting in irreducible representations of this algebra. Thus, we have to classify irreducible representations of the algebra (25).

Let us work in the eigenbasis $\{|z\rangle\}$ of the operator $Z$,

$$
Z|z\rangle=z|z\rangle
$$

It follows from Eqs. (25a), (25b), that $X_{+}, X_{-}$raise and lower the eigenvalue $z$ by one,

$$
X_{+}|z\rangle=x_{+}(z)|z+1\rangle, \quad X_{-}|z\rangle=x_{-}(z)|z-1\rangle
$$

Hence, in an irreducible representation, the eigenvectors of $Z$ may be labeled by an integer $k$, and in this basis one has

$$
\begin{gathered}
Z=\sum_{k}(k+\gamma)|k\rangle\langle k| \\
X_{+}=\sum_{k} x_{k}|k+1\rangle\langle k| \\
X_{-}=\sum_{k} x_{k}^{*}|k\rangle\langle k+1|,
\end{gathered}
$$

where $\gamma$ is a real parameter characteristic to the irreducible representation. Relation (25c) gives a recursion equation for the coefficients $x_{k}$. Let us consider the lower sign in Eq. (25c), then

$$
(\lambda+\mu)\left|x_{k}\right|^{2}-(\lambda-\mu)\left|x_{k-1}\right|^{2}=2 \mu .
$$

The solution of this equation is

$$
\left|x_{k}\right|^{2}=1+C \alpha^{k}
$$

where

$$
\alpha=\frac{\lambda-\mu}{\lambda+\mu}
$$

and $C$ is an arbitrary constant. Taking into account that $|\alpha|<1$ and that $\left|x_{k}\right|^{2}>0$ we obtain three different possibilities (up to redefinitions of $k, C$ and $\alpha$ ):

a) $C=0$ and $k$ runs from $-\infty$ to $+\infty$; in this case $x_{k}=1$,

b) $C=-1$ and $k$ runs from 1 to $+\infty$; in this case $x_{k}=\sqrt{1-\alpha^{k}}$,

c) $C>0$ and $\alpha>0$ and $k$ runs from $-\infty$ to $+\infty$.

One may give the following geometric interpretation to these three types of solutions. Let us consider them as axially symmetric fuzzy manifolds where the role of coordinates is 
played by covariant coordinates $X_{+}, X_{-}$and $Z$. This kind of interpretation is quite common in $\mathrm{NC}$ gauge theories; later it will be supported by the study of the spectrum of small perturbations. The case (a) corresponds just to the original fuzzy cylinder (see Fig. 1a)). The case $(b)$ corresponds to the semi-infinite fuzzy cylinder ("fuzzy fingerstall") of variable radius, starting at $z=\gamma$ and oriented towards positive values of $z$ (see Fig. 1b)). Finally, the case (c) corresponds to an infinite tube whose radius tends to unity as $z \rightarrow+\infty$ and becomes infinite as $z \rightarrow-\infty$ (see Fig. 1c)). It is straightforward to check that the topological charge

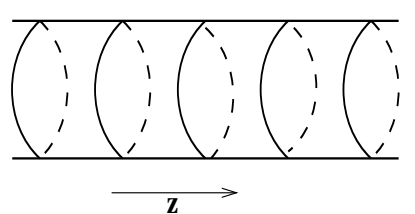

a

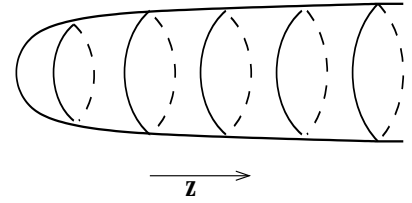

$\mathrm{b}$

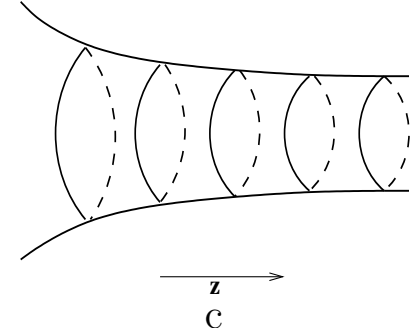

Figure 1: Geometrical interpretation of the three types of solutions to BPS equations (25) a) fuzzy cylinder, b) fuzzy fingerstall, c) infinitely expanding tube.

$Q$ is equal to zero in the first case, equal to one in the case $(b)$ and is ill-defined in the case (c). We will not consider solutions of the third type in this paper.

The solution corresponding to a domain wall between $U(1)$ and $U(2)$ gauge theories is a direct sum of representations $(a)$ and $(b)$,

$$
\begin{aligned}
& Z=\sum_{n=-\infty}^{0} n|n\rangle\left\langle n\left|+\sum_{n=1}^{\infty} n\right| 2 n-1\right\rangle\left\langle 2 n-1\left|+\sum_{n=1}^{\infty}(n+\gamma)\right| 2 n\right\rangle\langle 2 n| \\
& X_{+}=\sum_{n=-\infty}^{0}|n+1\rangle\left\langle n\left|+\sum_{n=1}^{\infty}\right| 2 n+1\right\rangle\left\langle 2 n-1\left|+\sum_{n=1}^{\infty} \sqrt{1-\alpha^{n}}\right| 2 n+2\right\rangle\langle 2 n| .
\end{aligned}
$$

This domain wall has a simple geometrical interpretation: it describes a parallel system of an infinite fuzzy cylinder and a half-infinite fuzzy fingerstall (see Fig. 2). The topological charge $Q$ of this solution is equal to unity, as expected. Without loss of generality we dropped in Eq. (26a) the possibility of the overall shift of $Z$ by a $c$-number. Parameter $\gamma$, as we will show later, characterizes the position of the wall along $z$-coordinate. The energy of the wall is

$$
\mathcal{E}=\frac{\pi m \rho}{2 g^{2} l^{2}}
$$




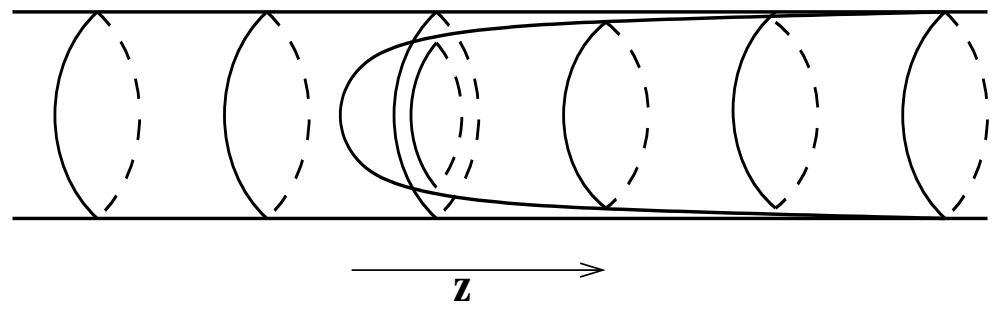

Figure 2: Geometrical interpretation of the domain wall: fingerstall inserted into the fuzzy cylinder.

Its width $\delta z$ can be estimated as the size of the region along $z$-direction, where exact solution (26) differs considerably from its asymptotic form (17). Then, restoring the dimensionality, one has

$$
\delta z=\frac{l}{|\ln \alpha|} \approx \frac{1}{m} \text { at } m \ll \frac{1}{l} .
$$

Note, that as $m$ tends to zero, the width of the wall becomes infinite. This justifies our previous claim that in NC pure Yang-Mills theory, the wall tends to dissolve. Thus, we keep $m \neq 0$. In the commutative limit $l \rightarrow 0$ the energy of the wall diverges, while its width stays finite. Another interesting limit is that of the NC plane. It corresponds to $\rho \rightarrow \infty$, $l \rightarrow 0$, and $\vartheta=\rho l$ fixed. In this regime the energy (27) diverges as $\rho^{3}$, which means that our solution does not correspond to a domain wall of finite tension on the NC plane.

The solution (26) is easy to generalize to $\left(U\left(\mathcal{N}_{1}\right)-U\left(\mathcal{N}_{2}\right)\right)$ domain wall with $\mathcal{N}_{1}<\mathcal{N}_{2}$. One simply takes the direct sum of $\mathcal{N}_{1}$ representations of type $(a)$ (cylinders) and $\mathcal{N}_{2}-\mathcal{N}_{1}$ representations of type $(b)$ (fingerstalls). The antiwall (that is $\left(U\left(\mathcal{N}_{1}\right)-U\left(\mathcal{N}_{2}\right)\right.$ ) wall with $\mathcal{N}_{1}>\mathcal{N}_{2}$ ) is the direct sum of irreducible representations of the algebra defined by relations (25) with upper sign in (25c). A system of a wall and an antiwall will be studied in detail in section 5 .

\section{Scalars and fermions in the domain wall background}

\subsection{Adjoint scalar}

To figure out the properties of the domain wall solution constructed in the previous section, let us consider a Hermitian adjoint scalar field $\phi$ in the $(U(1)-U(2))$ domain wall background. 
The action is

$$
S_{\phi}=2 \pi \rho l \int d t \operatorname{Tr}\left(\frac{1}{2}\left(D_{0} \phi\right)^{2}+\frac{1}{2 \rho^{2} l^{2}}\left([\mathbf{Z}, \phi]^{2}+[\mathbf{X}, \phi]^{2}+[\mathbf{Y}, \phi]^{2}\right)-\frac{1}{2} m_{\phi}^{2} \phi^{2}\right) .
$$

After redefinition (16) one obtains

$$
S_{\phi}=\frac{\pi l}{\rho} \int d t \operatorname{Tr}\left(\rho^{2}\left(D_{0} \phi\right)^{2}+[Z, \phi]^{2}-\lambda^{2}\left|\left[X_{+}, \phi\right]\right|^{2}-\mu_{\phi}^{2} \phi^{2}\right) .
$$

where $\mu_{\phi}=m_{\phi} \rho$. Equations for normal modes following from Eq. (29) are

$$
\rho^{2} \omega^{2} \phi=[Z,[Z, \phi]]+\frac{\lambda^{2}}{2}\left[X_{-},\left[X_{+}, \phi\right]\right]+\frac{\lambda^{2}}{2}\left[X_{+},\left[X_{-}, \phi\right]\right]+\mu_{\phi}^{2} \phi,
$$

where operators $Z, X_{+}$are given by (26). It is convenient to decompose the Hilbert space $H$ into direct sum $H=H_{1} \oplus H_{2}$ of two Hilbert spaces with bases

$$
\left|c_{n}\right\rangle= \begin{cases}|n\rangle & n \leq 0 \\ |2 n-1\rangle & n \geq 1\end{cases}
$$

and

$$
\left|h_{p}\right\rangle=|2 p\rangle, \quad p \geq 1
$$

respectively. Background operators (26) take the following form

$$
\begin{aligned}
& Z=\sum_{n=-\infty}^{\infty} n\left|c_{n}\right\rangle\left\langle c_{n}\left|+\sum_{p=1}^{\infty}(p+\gamma)\right| h_{p}\right\rangle\left\langle h_{p}\right| \\
& X_{+}=\sum_{n=-\infty}^{\infty}\left|c_{n+1}\right\rangle\left\langle c_{n}\left|+\sum_{p=1}^{\infty} \sqrt{1-\alpha^{p}}\right| h_{p+1}\right\rangle\left\langle h_{p}\right| .
\end{aligned}
$$

These operators are block-diagonal in the sense that they map $H_{i}$ to $H_{i}(i=1,2)$. It is convenient to decompose the field $\phi$ as

$$
\phi=\sum \phi_{n m}\left|c_{n}\right\rangle\left\langle c_{m}\left|+\sum \varphi_{p q}\right| h_{p}\right\rangle\left\langle h_{q}\right|+\sum\left(\chi_{n p}\left|c_{n}\right\rangle\left\langle h_{p}\left|+\chi_{n p}^{*}\right| h_{p}\right\rangle\left\langle c_{n}\right|\right)
$$

so that equations for different components $\phi_{n m}, \varphi_{p q}$ and $\chi_{n p}$ decouple. One immediately notices that the indices $p, q$ are greater than zero, so nonvanishing matrix elements of the operators $\varphi$ and $\chi$ have at least one positive index. This implies that the modes described by these operators live in the region $z>0$ and cannot penetrate into the region $z \rightarrow-\infty$. Let us study the three types of modes entering the decomposition (32) separately. 
Equations for the $\phi_{n m}$-components in the background (31) exactly coincide with equations for the scalar field on the fuzzy cylinder (9). Thus, excitations of this type do not feel the presence of the fingerstall and freely propagate from one end of the cylinder to the other.

Equations for the $\varphi_{p q}$-components have the following form,

$$
\begin{aligned}
\rho^{2} \omega^{2} \varphi_{p q} & =\left((p-q)^{2}+\mu_{\phi}^{2}+2 \lambda^{2}\left(1-\frac{\alpha^{p}+\alpha^{p-1}+\alpha^{q}+\alpha^{q-1}}{4}\right)\right) \varphi_{p q} \\
& -\lambda^{2} \sqrt{\left(1-\alpha^{p}\right)\left(1-\alpha^{q}\right)} \varphi_{p+1, q+1}-\lambda^{2} \sqrt{\left(1-\alpha^{p-1}\right)\left(1-\alpha^{q-1}\right)} \varphi_{p-1, q-1} .
\end{aligned}
$$

Let us perform Kaluza-Klein decomposition in analogy to the case of free scalar field considered in section 2. Namely, after fixing the number of the Kaluza-Klein mode, $p-q=N$, we obtain the following recursion relation for $\varphi_{q} \equiv \varphi_{q+N, q}$,

$$
\begin{aligned}
\rho^{2} \omega^{2} \varphi_{q} & =\left(N^{2}+\mu_{\phi}^{2}+2 \lambda^{2}\left(1-\frac{\alpha^{N+q}+\alpha^{N+q-1}+\alpha^{q}+\alpha^{q-1}}{4}\right)\right) \varphi_{q} \\
& -\lambda^{2} \sqrt{\left(1-\alpha^{N+q}\right)\left(1-\alpha^{q}\right)} \varphi_{q+1}-\lambda^{2} \sqrt{\left(1-\alpha^{N+q-1}\right)\left(1-\alpha^{q-1}\right)} \varphi_{q-1} .
\end{aligned}
$$

At large $q$, the general solution of this equation is

$$
\varphi_{q}=A \mathrm{e}^{i k l q}+B \mathrm{e}^{-i k l q},
$$

where the wave vector $k$ and frequency $\omega$ are related by Eq. (8). To relate the coefficients $A$ and $B$ let us note that equation (33) leads to the conservation of "current",

$$
J_{q+1}-J_{q}=0
$$

with

$$
J_{q}=i \sqrt{\left(1-\alpha^{N+q}\right)\left(1-\alpha^{q}\right)}\left(\varphi_{q+1}^{*} \varphi_{q}-\varphi_{q}^{*} \varphi_{q+1}\right) .
$$

Equation (33) with $q=1$ implies that

$$
\varphi_{2}=c \varphi_{1}
$$

with real coefficient of proprtionality $c$. So $J_{1}=0$ and, as a consequence, we obtain that $J_{q}=0$ for all $q$. Then the coefficients in Eq. (34) have equal absolute values,

$$
|A|=|B| .
$$

The asymptotic solution (34) describes two waves with equal amplitudes propagating in the opposite directions. In other words, excitations $\varphi_{p q}$ experience total reflection from the 
domain wall. Note that these modes do not feel the presence of the fuzzy cylinder and live entirely on the fingerstall.

Modes $\chi_{n p}$ obey the following equation,

$$
\begin{aligned}
\rho^{2} \omega^{2} \chi_{n p}=\left((n-p-\gamma)^{2}+\mu_{\phi}^{2}+2 \lambda^{2}(1\right. & \left.\left.-\frac{\alpha^{p}+\alpha^{p-1}}{4}\right)\right) \chi_{n p} \\
& \quad-\lambda^{2} \sqrt{1-\alpha^{p-1}} \chi_{n-1, p-1}-\lambda^{2} \sqrt{1-\alpha^{p}} \chi_{n+1, p+1} .
\end{aligned}
$$

By fixing $n-p=N$ and substituting $\chi_{p} \equiv \chi_{p+N, p}$ one obtains

$$
\begin{aligned}
\rho^{2} \omega^{2} \chi_{p}=\left((N-\gamma)^{2}+2 \lambda^{2}\left(1-\frac{\alpha^{p}+\alpha^{p-1}}{4}\right)\right) & \chi_{p} \\
& -\lambda^{2} \sqrt{1-\alpha^{p-1}} \chi_{p-1}-\lambda^{2} \sqrt{1-\alpha^{p}} \chi_{p+1} .
\end{aligned}
$$

The analysis similar to that for modes $\varphi$ demonstrates that solutions of this equation are waves which experience total reflection from the domain wall. Their dispersion relation is

$$
\omega^{2}=\frac{(N-\gamma)^{2}}{\rho^{2}}+\left(\frac{2}{l} \sin \frac{k l}{2}\right)^{2}+m_{\phi}^{2} .
$$

Let us comment on this result. First, if the parameter $\gamma$ is not integer, spectrum of the off-diagonal modes $\chi_{n p}$ differs from that of diagonal modes $\phi_{n m}$ and $\varphi_{p q}$. As $z \rightarrow+\infty$, the $\chi$ and $(\phi, \varphi)$ modes correspond to off-diagonal and diagonal components of the adjoint $U(2)$ field, respectively. Difference in their dispersion relations means that in the case of non-integer $\gamma$ the gauge group $U(2)$ is broken down to $U(1) \times U(1)$ at $z \rightarrow+\infty$. This effect is nothing else than spontaneous breaking of the gauge symmetry by a nontrivial Wilson line on the cylinder. Indeed, from (13c) and (26a) we see that

$$
A_{\theta}=\left(\begin{array}{ll}
0 & 0 \\
0 & \gamma
\end{array}\right), \quad z \rightarrow+\infty \text {. }
$$

The value of the Wilson line around the cylinder is equal to $e^{2 \pi i \gamma} \neq 1$ if $\gamma$ is not integer. Thus, $A_{\theta}$ cannot be removed by a gauge transformation in this case and breaks the gauge symmetry. Conversely, if $\gamma$ is integer, $A_{\theta}$ is a pure gauge as $z \rightarrow+\infty$, and the symmetry $U(2)$ is unbroken in the $z \rightarrow+\infty$ asymptotics. From now on we consider integer values of $\gamma$ only.

Let us see now that the parameter $\gamma$ is related to the position of the domain wall. Equation (35) implies that the genuine Kaluza-Klein number of a $\chi$ excitation which characterizes its energy is $N_{0}=N-\gamma$. The expression for $N_{0}$ th KK excitation in the operator form is

$$
\chi^{\left(N_{0}\right)}=\sum_{p=1}^{\infty} \chi_{p}^{\left(N_{0}\right)}\left|c_{\gamma+N_{0}+p}\right\rangle\left\langle h_{p}\right|
$$


The operator $\chi^{\left(N_{0}\right)}\left(\chi^{\left(N_{0}\right)}\right)^{\dagger}$ acts diagonally in $H_{1}$ and annihilates all vectors with

$$
n<n_{\min } \equiv N_{0}+\gamma+1 \text {. }
$$

Hence, the $\chi$-wave is reflected from a point with $z$-coordinate $n_{\min }$. For a given KK mode this coordinate depends additively on $\gamma$, implying that this parameter is natural to interpret as the position of the wall ${ }^{5}$.

Let us summarize our results on the spectrum of the adjoint field in the presence of the domain wall. In the region $z \rightarrow+\infty$ there are four (real) modes which constitute adjoint representation of $U(2)$. Their dispersion relation is given by Eq. (8) which reduces to the standard dispersion relation on the cylinder in the long wavelength regime. Three of these modes are reflected from the wall while one freely propagates to the region $z \rightarrow-\infty$. A notable fact is that the latter mode does not decouple from the other modes in the region $z \rightarrow+\infty$ : in terms of $U(2)$ group it has the form $\left(\begin{array}{ll}\phi & 0 \\ 0 & 0\end{array}\right)$, and couples to other modes due to gauge interactions. These results can be straightforwardly generalized to the case of the $\left(U\left(\mathcal{N}_{1}\right)-U\left(\mathcal{N}_{2}\right)\right)$ domain wall with $\mathcal{N}_{1}<\mathcal{N}_{2}$. In that case there are $\mathcal{N}_{1}^{2}$ modes in the adjoint of $U\left(\mathcal{N}_{1}\right)$ group freely propagating along the cylinder and $\left(\mathcal{N}_{2}^{2}-\mathcal{N}_{1}^{2}\right)$ modes exhibiting reflection from the domain wall.

The analysis of gauge field perturbations in the domain wall background is less transparent because of mixing between different components and the necessity to implement the Gauss' constraint. We do not give the details here, and only states that the qualitative picture described in the previous paragraph holds for the gauge field perturbations as well. Namely, the $U\left(\mathcal{N}_{1}\right)$ gauge fields propagate freely along the entire cylinder; in the region $z \rightarrow+\infty$ they become a part of $U\left(\mathcal{N}_{2}\right)$ gauge multiplet; the rest of the $U\left(\mathcal{N}_{2}\right)$ gauge fields live only in the region on the right and are totally reflected from the wall.

\subsection{Fundamental fermion}

To study fermions in the background of the domain wall, we have to define the Dirac operator on the fuzzy cylinder. To this end let us consider possible choices of the Dirac Hamiltonian on the commutative cylinder. The simplest one is

$$
\tilde{D}=i \sigma_{2} \partial_{z}-\frac{i}{\rho} \sigma_{3} \partial_{\theta}+\sigma_{1} m_{f}
$$

\footnotetext{
${ }^{5}$ Strictly speaking, the above argument is not rigorous. The reason is that bilinear combinations of adjoint fields are again adjoints and are not gauge invariant, while in NC theory gauge transformations of adjoints include change of coordinates. Our conclusion that $\gamma$ characterizes the position of the domain wall will be confirmed by the consideration of a fundamental field in the next subsection.
} 
where $m_{f}$ is the fermion mass and $\sigma_{i}$ are Pauli matrices. This operator is not convenient for our purposes, because natural derivative operators on the fuzzy cylinder are $x \partial_{z}$ and $y \partial_{z}$ rather than $\partial_{z}$ (see Eqs. (5a), (5b) $)^{6}$. So, we will work with the following unitary equivalent operator

$$
D=S^{+} \tilde{D} S
$$

where

$$
S=\left(\begin{array}{cc}
e^{i \theta / 2} & 0 \\
0 & e^{-i \theta / 2}
\end{array}\right)
$$

Explicitly, one has

$$
D=\frac{1}{\rho}\left(-i\left(\sigma_{1} y-\sigma_{2} x\right) \partial_{z}-i \sigma_{3} \partial_{\theta}+\frac{1}{2}+\left(\sigma_{1} x+\sigma_{2} y\right) m_{f}\right) .
$$

Let us note that due to the form (36) of the unitary transformation $S$, periodic boundary conditions for the operator $D$ correspond to anti-periodic boundary conditions for $\tilde{D}$ and vice versa. This is not problematic, as one may consider both periodic and anti-periodic spinors on the cylinder.

Noncommutative counterpart of the operator (37) can be naturally defined in terms of its action on a spinor field $\psi$ as (cf. Eqs. (4), (5))

$$
D \psi=\frac{1}{\rho l}\left(\sigma_{1}[\mathbf{x}, \psi]+\sigma_{2}[\mathbf{y}, \psi]+\sigma_{3}[\mathbf{z}, \psi]\right)+\frac{1}{2 \rho} \psi+\frac{m_{f}}{2 \rho}\left(\sigma_{1}\{\mathbf{x}, \psi\}+\sigma_{2}\{\mathbf{y}, \psi\}\right) .
$$

The operator ordering in the mass term in Eq. (38) is chosen somewhat arbitrarily; a different choice would not alter the results of the analysis below but would make calculations more cumbersome. The spectrum of the operator (38) is

$$
\omega^{2}=\frac{(N+1 / 2)^{2}}{\rho^{2}}+\left(\frac{2}{l} \sin \frac{k l}{2}\right)^{2}+\left(m_{f} \cos \frac{k l}{2}\right)^{2}
$$

${ }^{6}$ Nevertheless, one could proceed with the operator $\tilde{D}$ and rewrite the term $i \sigma_{2} \partial_{z} \psi$ as

$$
i \frac{\sigma_{2}}{\rho^{2}}\left(x \cdot x \partial_{z} \psi+y \cdot y \partial_{z} \psi\right)
$$

Noncommutative counterpart of this expression would be

$$
\frac{\sigma_{2}}{\rho^{2} l}(\mathbf{x}[\mathbf{y}, \psi]-\mathbf{y}[\mathbf{x}, \psi])=\frac{\sigma_{2}}{\rho^{2} l}(\mathbf{x} \psi \mathbf{y}-\mathbf{y} \psi \mathbf{x})
$$

This operator leads to the second order recursion equations and its spectrum suffers from doubling of fermion species, resembling that occurring in the lattice field theory. 
where $N$ is the Kaluza-Klein number and $k$ is the wave vector. Two comments on this dispersion relation are in order. First, the appearance of $(N+1 / 2)$ instead of $N$ in the first term of Eq. (39) is not unexpected, since as discussed above we are effectively considering anti-periodic fermions. The dependence on $k$ in the third (mass) term is more surprising. Interestingly, the same dependence appears in the dispersion relation for the field $A_{\rho}$ defined by Eqs. (13a), (13b) when the term (20) is added to the gauge action.

The Dirac Hamiltonian for fermion in the fundamental representation of the gauge group is obtained from Eq. (38) via substitution

$$
\begin{aligned}
& {[\mathbf{x}, \psi] \longrightarrow \mathbf{X} \psi-\psi \mathbf{x}} \\
& \{\mathbf{x}, \psi\} \longrightarrow \mathbf{X} \psi+\psi \mathbf{x}
\end{aligned}
$$

and similarly for other coordinates. In the domain wall background (26), it is convenient to decompose the fermion field in the following way (cf. Eq. (32))

$$
\psi=\sum_{n, m=-\infty}^{\infty} \psi_{n m}\left|c_{n}\right\rangle\left\langle m\left|+\sum_{p=1, n=-\infty}^{\infty} \eta_{p n}\right| h_{p}\right\rangle\langle n|
$$

Equations for the $\psi_{n m}$-components are identical to those obtained in the case of fuzzy cylinder without the domain wall, and the corresponding branch of the spectrum is given by Eq. (39). Thus, these modes do not feel the presence of the domain wall at all. For spinors $\eta_{p n}$ we obtain the following equations,

$$
\omega \rho\left(\begin{array}{c}
\eta_{p n}^{1} \\
\eta_{p n}^{2}
\end{array}\right)=\left(\begin{array}{c}
\left(p-n+\gamma+\frac{1}{2}\right) \eta_{p n}^{1}+\left(\lambda+\frac{\mu_{f}}{2}\right) \sqrt{1-\alpha^{p}} \eta_{p+1, n}^{2}-\left(\lambda-\frac{\mu_{f}}{2}\right) \eta_{p, n-1}^{2} \\
\left(\lambda+\frac{\mu_{f}}{2}\right) \sqrt{1-\alpha^{p-1}} \eta_{p-1, n}^{1}-\left(\lambda-\frac{\mu_{f}}{2}\right) \eta_{p, n+1}^{1}-\left(p-n+\gamma-\frac{1}{2}\right) \eta_{p n}^{2}
\end{array}\right)
$$

where $\mu_{f}=m_{f} \rho$. After substituting $\eta_{p}^{1} \equiv \eta_{p, p+N}^{1}, \eta_{p}^{2} \equiv \eta_{p, p+N-1}^{2}$, equations along diagonals $n-p=N$ take the form

$$
\omega \rho\left(\begin{array}{c}
\eta_{p}^{1} \\
\eta_{p}^{2}
\end{array}\right)=\left(\begin{array}{c}
\left(-N+\gamma+\frac{1}{2}\right) \eta_{p}^{1}+\left(\lambda+\frac{\mu_{f}}{2}\right) \sqrt{1-\alpha^{p}} \eta_{p+1}^{2}-\left(\lambda-\frac{\mu_{f}}{2}\right) \eta_{p}^{2} \\
\left(\lambda+\frac{\mu_{f}}{2}\right) \sqrt{1-\alpha^{p-1}} \eta_{p-1}^{1}-\left(\lambda-\frac{\mu_{f}}{2}\right) \eta_{p}^{1}-\left(-N+\gamma+\frac{1}{2}\right) \eta_{p}^{2}
\end{array}\right) .
$$

Since $n=p+N>N$, a KK mode with fixed $N$ does not penetrate into the region $z \rightarrow-\infty$ and thus is reflected from the domain wall. In this respect the situation is similar to the case of the adjoint scalar considered in the previous subsection.

Let us find now a zero mode localized on the domain wall. By zero mode we understand a mode which is annihilated by the transverse part of the Dirac Hamiltonian. In other words, 
it obeys the following equations

$$
\begin{aligned}
& \left(\lambda+\frac{\mu_{f}}{2}\right) \sqrt{1-\alpha^{p-1}} \eta_{p-1}^{1}-\left(\lambda-\frac{\mu_{f}}{2}\right) \eta_{p}^{1}=0 \\
& \left(\lambda+\frac{\mu_{f}}{2}\right) \sqrt{1-\alpha^{p}} \eta_{p+1}^{2}-\left(\lambda-\frac{\mu_{f}}{2}\right) \eta_{p}^{2}=0
\end{aligned}
$$

Equation (40) with $p=1$ implies that $\eta_{1}^{1}=0$, and as a consequence

$$
\eta_{p}^{1}=0
$$

for all $p$. From Eq. (41) one obtains the following solution for $\eta_{p}^{2}$-components

$$
\eta_{p}^{2}=C \beta^{p-1} \prod_{j=1}^{p-1}\left(1-\alpha^{j}\right)^{-\frac{1}{2}}
$$

where

$$
\beta=\frac{\lambda-\mu_{f} / 2}{\lambda+\mu_{f} / 2}
$$

and $C$ is a normalization constant. This solution is well-behaved at large $p$ for $\mu_{f}>0$. Note that zero mode (42), (43) is chiral

$$
\sigma_{3} \eta=-\eta
$$

A zero mode with opposite chirality is localized on the antiwall configuration.

Let us work out the profile of zero mode along $z$-direction. This profile is gauge invariant and can be regardedd as a probe of the domain wall shape. We assume $\mu, \mu_{f} \ll \lambda$. First, let us note that the energy of zero mode on the $N$-th diagonal is given by

$$
\omega=\frac{N-\gamma-1 / 2}{\rho} .
$$

Thus, the genuine Kaluza-Klein number of this mode is $N_{0}=N-\gamma-1$. From Eq. (43) one obtains the following gauge invariant density

$$
\eta^{\dagger} \eta=\sum_{p=1}^{\infty}\left(\eta_{p}^{2}\right)^{2}\left|N_{0}+\gamma+p\right\rangle\left\langle N_{0}+\gamma+p\right| .
$$

Its Weyl symbol is shown in Fig. 3. This density concentrates near $p=p_{\max }$ with $p_{\max }$ determined by

$$
1-\alpha^{p_{\max }}=\beta^{2}
$$

or, explicitly,

$$
p_{\max }=\frac{\lambda}{2 \mu} \ln \frac{\lambda}{2 \mu_{f}}
$$




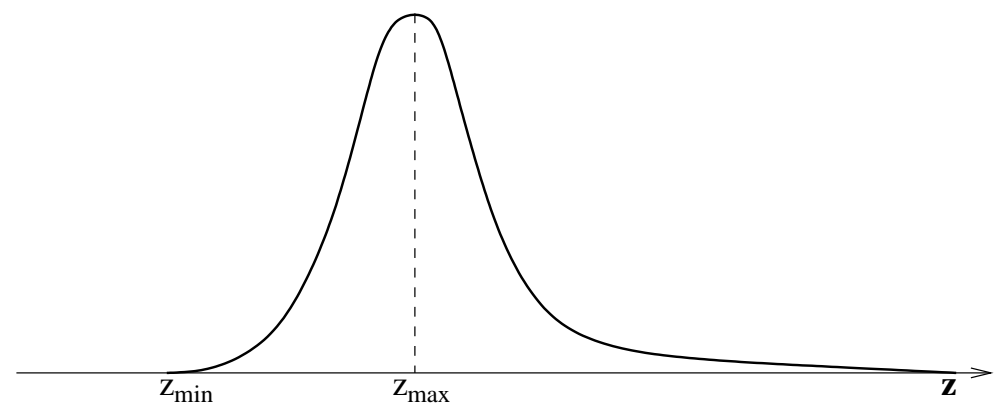

Figure 3: Gauge invariant profile of zero fermion mode localized on the domain wall.

Coefficients entering Eq. (44) may be approximated in the vicinity of $p_{\max }$ as follows,

$$
\left(\eta_{p}^{2}\right)^{2}=\tilde{C}^{2} \exp \left(-\frac{2 \mu_{f} \mu}{\lambda^{2}}\left(p-p_{\max }\right)^{2}\right)
$$

where $\tilde{C}$ is a constant. From Eqs. (44) and (46), one infers the following properties of the Weyl symbol $\widetilde{\eta^{\dagger} \eta}(z)$ in terms of physical parameters: it is equal to zero at $z<z_{\text {min }}$, where

$$
z_{\min }=l \gamma+l N_{0}
$$

peaks in the vicinity of

$$
z_{\max }=\frac{1}{m} \ln \frac{1}{2 m_{f} l}+l \gamma+l N_{0}
$$

where it has the form

$$
\widetilde{\eta^{\dagger} \eta}(z)=\tilde{C}^{2} \exp \left(-m_{f} m\left(z-z_{\max }\right)^{2}\right),
$$

and falls off exponentially at $z \rightarrow+\infty$. The coordinates $z_{\min }$ and $z_{\max }$ can serve as two alternative definitions of the position of the domain wall. If $N_{0}$ is not too large, the last terms in Eqs. (47), (48) can be neglected. Then, $z_{\text {min }} \approx l \gamma$ coincides with our previous naive estimate of the domain wall position, based on gauge dependent quantities (see discussion after Eq. (35)).

On the other hand, $z_{\max }$ is more appropriate for the definition of the position of the wall, if one considers $\widetilde{\eta^{\dagger} \eta}(z)$ as the gauge independent shape of the domain wall seen by the zero fermionic mode. The disadvantage of this definition is that it depends on the mass of the fermion, but it is problematic to provide a probe independent meaning to the notion of the domain wall shape. Assuming $m_{f} \approx m$, one obtains from Eqs. (48), (49) the following estimates for the position $z_{\max }$ and width $\delta z$ of the domain wall

$$
z_{\max } \approx \frac{1}{m} \ln \frac{1}{2 m l}+l \gamma, \quad \delta z \approx \frac{1}{m} .
$$


Let us stress once more that these estimates refer to the domain wall as seen by the zero fermionic mode, and other probes may, in principle, give other results. The estimate for the width agrees with that deduced from the (gauge dependent) profile of the domain wall, see Eq. (28).

\section{Wall-antiwall system}

Similarly to the domain wall solution, it is convenient to describe the wall-antiwall configuration in terms of operators acting on a direct sum of Hilbert spaces $H=H_{1} \oplus H_{2}$. Subspaces $H_{1}$ and $H_{2}$ emerging in the case of the wall-antiwall system are spanned by the following systems of basis vectors

$$
\begin{aligned}
& \left|c_{n}\right\rangle=|n\rangle, \quad n \leq 0 \\
& \left|h_{p}\right\rangle=|p\rangle, \quad p \geq 1 .
\end{aligned}
$$

In these notations the wall-antiwall field has the following form

$$
\begin{aligned}
& Z=\sum_{n=-\infty}^{0}\left(n+\gamma_{1}\right)\left|c_{n}\right\rangle\left\langle c_{n}\left|+\sum_{p=1}^{\infty}\left(p+\gamma_{2}\right)\right| h_{p}\right\rangle\left\langle h_{p}\right| \\
& X_{+}=\sum_{n=-\infty}^{0} \sqrt{1-\alpha^{-n}}\left|c_{n+1}\right\rangle\left\langle c_{n}\left|+\sum_{p=1}^{\infty} \sqrt{1-\alpha^{p}}\right| h_{p+1}\right\rangle\left\langle h_{p}\right|
\end{aligned}
$$

This may be viewed as the union of two fingerstalls. Two physically different situations occur depending on the value of the parameter $\Delta \gamma \equiv \gamma_{2}-\gamma_{1}$ : for $\Delta \gamma>0$ the two fingerstalls intersect (see Fig. 4a) and, as we will see below, $U(2)$ gauge theory emerges in the region between the walls, while for $\Delta \gamma<0$ the fingerstalls are disconnected (Fig. 4b).

Due to the direct sum structure of Eqs. (51), they describe an exact solution of field equations. This is somewhat unexpected, because it implies that there is no attraction between the wall and antiwall. On the other hand, configuration (51) belongs to the trivial topological sector (it is straightforward to check that its topological charge $Q$ defined in (24) is equal to zero), and, consequently, it is expected to be unstable. We will show this explicitly later on.

To justify our interpretation of the two-fingerstall configuration shown in Fig. 4a as a wall-antiwall system, let us address a question of the restoration of non-Abelian symmetry in the region where the fingerstalls overlap. When the size of this region is large,

$$
\Delta \gamma \gg \frac{1}{|\ln \alpha|}
$$




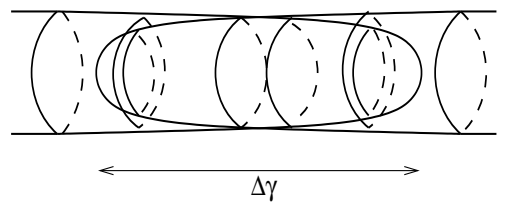

a

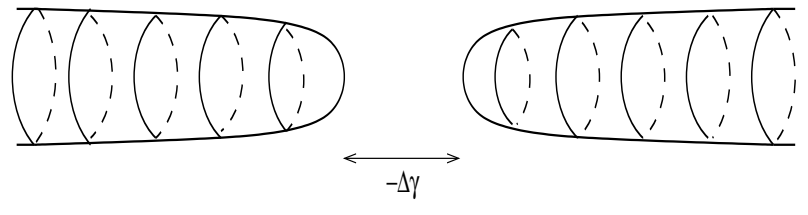

$\mathrm{b}$

Figure 4: Two possibilities for the non-BPS solution of two fingerstalls: a) Two regions with $U(1)$ theories separated by a region with $U(2)$ theory; b) Two disjoint regions with $U(1)$ theories

we expect $U(2)$ gauge symmetry to emerge in the overlap region. Direct analysis of gauge field fluctuations in the background (51) is rather cumbersome. More illuminating, as in the case of the single domain wall, is to consider an adjoint scalar field $\phi$. It obeys Eq. (30) with the background given by Eqs. (51). For simplicity we consider massless field, $m_{\phi}^{2}=0$. In analogy to the single domain wall case, we perform the decomposition (32). Dispersion relations for modes $\phi_{n m}$ and $\varphi_{p q}$ are again given by Eq. (8). These are modes living on each of the fingerstalls separately. Modes $\phi_{n m}$ do not feel the presence of the domain wall, but are reflected back from the antiwall, while modes $\varphi_{p q}$ do not feel the antiwall but are reflected by the wall. The same qualitative picture is valid for the modes of the gauge fields responsible for $U(1)$ gauge symmetry in the asymptotic regions $z \rightarrow-\infty$ and $z \rightarrow+\infty$. This means that the gauge fields from $z \rightarrow-\infty$ region cannot penetrate into $z \rightarrow+\infty$ region, and vice versa.

Modes $\phi_{n m}$ and $\varphi_{p q}$ correspond to diagonal elements of the $U(2)$ multiplet in the region between the walls. Off-diagonal components of the multiplet should come from the $\chi_{n p}$ sector. Equation for these modes reads

$$
\begin{aligned}
\rho^{2} \omega^{2} \chi_{n p}= & \left((p-n-\Delta \gamma)^{2}+2 \lambda^{2}\left(1-\frac{\alpha^{p}+\alpha^{p-1}+\alpha^{-n}+\alpha^{-n+1}}{4}\right)\right) \chi_{n p} \\
& -\lambda^{2} \sqrt{\left(1-\alpha^{p-1}\right)\left(1-\alpha^{-n+1}\right)} \chi_{n-1, p-1}-\lambda^{2} \sqrt{\left(1-\alpha^{p}\right)\left(1-\alpha^{-n}\right)} \chi_{n+1, p+1} .
\end{aligned}
$$

Performing the Kaluza-Klein decomposition, $p-n=N, \chi_{p+N, p} \equiv \chi_{p}$, one obtains

$$
\begin{aligned}
\rho^{2} \omega^{2} \chi_{p}=\left((N-\Delta \gamma)^{2}\right. & \left.+2 \lambda^{2}\left(1-\frac{\alpha^{p}+\alpha^{p-1}+\alpha^{N-p}+\alpha^{N-p+1}}{4}\right)\right) \chi_{p} \\
& -\lambda^{2} \sqrt{\left(1-\alpha^{p-1}\right)\left(1-\alpha^{N-p+1}\right)} \chi_{p-1}-\lambda^{2} \sqrt{\left(1-\alpha^{p}\right)(N-p)} \chi_{p+1} .
\end{aligned}
$$


The index $p$ in Eq. (53) runs from 1 to $N$ because $n \leq 0$. If the condition (52) is satisfied, low energy modes are those with

$$
\left|N_{0}\right|=|N-\Delta \gamma| \ll \Delta \gamma
$$

and their dispersion relation is approximately given by Eq. (8) (with $N_{0}$ instead of $N$ ). Strictly speaking, the wave vector $k$ of these modes is not arbitrary and takes discrete values determined by the boundary conditions at $p=1, N$. But the gap between two neighboring values is of order

$$
\Delta k \approx \frac{\pi}{l \Delta \gamma}
$$

and tends to zero as $\Delta \gamma \rightarrow \infty$. Thus, in the limit of large separation between the walls, the spectra of the off-diagonal and diagonal modes coincide, and $U(2)$ gauge symmetry emerges in the region between the walls.

Now, let us return to the question of stability of the wall-antiwall solution. As there is no force between the wall and antiwall, instability, if any, must reveal itself in the existence of a tachyonic mode in the spectrum of fluctuations of gauge fields about the wall-antiwall background. Clearly, this tachyon should carry indices corresponding to both fingerstalls comprising the solution (51). Thus, we consider fluctuations of the form

$$
Z=Z^{(0)}-a_{\theta}, \quad X_{+}=X_{+}^{(0)}+b_{+}
$$

where $Z^{(0)}, X_{+}^{(0)}$ are given by Eqs. (51), and $a_{\theta}, b_{+}$are off-diagonal

$$
\begin{aligned}
& a_{\theta}=\sum\left(a_{p n}\left|h_{p}\right\rangle\left\langle c_{n}\left|+a_{p n}^{*}\right| c_{n}\right\rangle\left\langle h_{p}\right|\right) \\
& b_{+}=\sum\left(b_{p n}\left|h_{p}\right\rangle\left\langle c_{n}\left|+c_{n p}\right| c_{n}\right\rangle\left\langle h_{p}\right|\right) .
\end{aligned}
$$

Insertion of these expressions into Eq. (21) yields quadratic energy functional for the fluctuations. The latter can be expressed as the sum of contributions coming from different diagonals $p-n-1=N$. The contribution due to the $N$ th diagonal is (we drop the overall 
dimensional prefactor $\frac{2 \pi}{g^{2} \rho l}$ in the energy)

$$
\begin{aligned}
\mathcal{E}_{N}=\sum_{p=1}^{p=N+1}\left(\left|(N-\Delta \gamma) b_{p}-\sqrt{1-\alpha^{N-p+1}} a_{p}+\sqrt{1-\alpha^{p-1}} a_{p-1}\right|^{2}\right. \\
+\left|(N-\Delta \gamma) c_{p}^{*}+\sqrt{1-\alpha^{p-1}} a_{p}-\sqrt{1-\alpha^{N-p+1}} a_{p-1}\right|^{2} \\
+\frac{\lambda^{2}}{2}\left|\sqrt{1-\alpha^{p-1}} c_{p}^{*}+\sqrt{1-\alpha^{N-p+1}} b_{p}-\sqrt{1-\alpha^{N-p}} c_{p+1}^{*}-\sqrt{1-\alpha^{p}} b_{p+1}\right|^{2} \\
+\frac{\mu^{2}}{2}\left|\sqrt{1-\alpha^{p-1}} c_{p}^{*}+\sqrt{1-\alpha^{N-p+1}} b_{p}+\sqrt{1-\alpha^{N-p}} c_{p+1}^{*}+\sqrt{1-\alpha^{p}} b_{p+1}\right|^{2} \\
\left.+\mu \lambda\left(\alpha^{p-1}+\alpha^{N-p+1}\right)\left|c_{p}\right|^{2}-\mu \lambda\left(\alpha^{p-1}+\alpha^{N-p+1}\right)\left|b_{p}\right|^{2}\right) .
\end{aligned}
$$

Notations in this expression are

$$
\begin{array}{ll}
b_{p}=b_{p,-N+p-1} & p=1,2, \ldots, N+1 \\
a_{p}=a_{p,-N+p} & p=1,2, \ldots, N \\
c_{p}=c_{-N+p, p-1} & p=2,3, \ldots, N .
\end{array}
$$

The expression (54) is rather lengthy, and we presented it just to demonstrate that the only negative contribution to the energy comes from the modes $b_{p}$. This suggests that these modes give the dominant contribution to the tachyon.

The following analysis is different for the two cases shown in Fig. 4. Let us consider first the case $\Delta \gamma<0$. We argue that in this case the lowest eigenvalue of the energy is given by the contribution of the zeroth diagonal. Indeed, modes along other diagonals have larger Kaluza-Klein energy (which is proportional to $(N-\Delta \gamma)^{2}$ and $N \geq 0$ ). This effect shifts upwards masses squared of these modes. Thus, let us study the case $N=0$. The expression (54) is greatly simplified yielding

$$
\mathcal{E}_{0}=\left((\Delta \gamma)^{2}-2 \mu \lambda\right)\left|b_{1}\right|^{2}
$$

We see that $b_{1}$ is an eigenmode with the frequency

$$
\omega^{2}=\frac{(\Delta \gamma)^{2}}{\rho^{2}}-\frac{m}{l}
$$

This means that at $\Delta \gamma<0$, the system is unstable, provided that $|\Delta \gamma|<\rho \sqrt{m / l}$, but becomes stable at large values of $|\Delta \gamma|$. This behavior is fairly natural, because when $\Delta \gamma<0$ 
and $|\Delta \gamma|$ is large, two fingerstalls comprising the wall-antiwall system do not intersect and are well separated (Fig. 4b).

Let us now turn to the case $\Delta \gamma>0$. If $\Delta \gamma$ satisfies Eq. (52), there is a large region of intersection of the two fingerstalls with $U(2)$ gauge theory inside (Fig. 4a). One expects the system to be able to roll down to either $U(1)$ or $U(2)$ vacuum on the whole cylinder. Instead of trying to find the tachyonic mode exactly, let us present an Ansatz which demonstrates that the energy functional given by Eq. (54) has negative directions. To this end, let us choose $N=\Delta \gamma$, take $a_{p}=0, c_{p}=0$ and rewrite the energy $\mathcal{E}_{N=\Delta \gamma}$ in the following form

$$
\begin{array}{r}
\mathcal{E}_{N=\Delta \gamma}=\sum_{p=1}^{N / 2} \frac{1}{2}\left((\lambda+\mu)^{2}\left|\alpha \sqrt{1-\alpha^{N-p+1}} b_{p}-\sqrt{1-\alpha^{p}} b_{p+1}\right|^{2}-4 \lambda \mu \alpha^{N-p+1}\left|b_{p}\right|^{2}\right) \\
+\sum_{p=N / 2+1}^{N+1} \frac{1}{2}\left((\lambda+\mu)^{2}\left|\sqrt{1-\alpha^{N-p+1}} b_{p}-\alpha \sqrt{1-\alpha^{p}} b_{p+1}\right|^{2}-4 \lambda \mu \alpha^{p-1}\left|b_{p}\right|^{2}\right) \\
-2 \lambda \mu\left(1-\alpha^{N / 2}\right)\left|b_{N / 2+1}\right|^{2} .
\end{array}
$$

Positive terms in eq. (55) can be set to zero by the following choice

$$
b_{p}= \begin{cases}\alpha^{p-1} \prod_{j=1}^{p-1} \sqrt{\frac{1-\alpha^{N-j+1}}{1-\alpha^{j}}} & p=1, \ldots, N / 2 \\ \alpha^{N+1-p} \prod_{j=1}^{p-1} \sqrt{\frac{1-\alpha^{N-j+1}}{1-\alpha^{j}}} & p=N / 2+1, \ldots, N+1 .\end{cases}
$$

This Ansatz for $b_{p}$ is a symmetric combination of two bell-shaped functions localized around $p_{\max }$ and $\left(N-p_{\max }\right)$, where $p_{\max }$ is given by Eq. (45) (with $\mu$ instead of $\mu_{f}$ ). Substitution of the Ansatz (56) into the energy functional (55) yields the following estimate for the tachyon energy

$$
\omega^{2}=-C \frac{4 \mu^{2}}{\rho^{2}} \alpha^{\Delta \gamma-2 p_{\max }}=-C m^{2} \mathrm{e}^{-m \Delta z},
$$

where $\Delta z=l\left(\Delta \gamma-2 p_{\max }\right)$ is the separation between the wall and antiwall, and $C$ is a coefficient of order one. We see that the tachyon mass is exponentially small when the walls are far away from each other.

This tachyon is not directly related to the distance between domain walls $\Delta \gamma$ which is an exact modulus of the wall-antiwall solution. One may guess that tachyon condensation leads to the change of the shapes of the walls. The natural candidates for the end points of the tachyon condensation are $U(1)$ and/or $U(2)$ vacua. We leave the study of this condensation for future. 


\section{$6 \quad M($ atrix) theory interpretation of the domain walls}

The purpose of this section is to suggest a way of embedding the domain wall solutions constructed in this paper into the matrix model of M-theory (see, e.g., Ref. [12] for a review of the M(atrix) model). Matrix model is supersymmetric quantum mechanics described by the following Lagrangian

$$
L=\frac{1}{2 R} \operatorname{Tr}\left\{\dot{\mathbf{X}}^{i} \dot{\mathbf{X}}^{i}+\frac{1}{2}\left[\mathbf{X}^{i}, \mathbf{X}^{j}\right]^{2}+(\text { fermions })\right\}
$$

where $\mathbf{X}^{i}(i=1, \ldots, 9)$ are real-valued $N \times N$ matrices subject to the constraint

$$
\left[\dot{\mathbf{X}}^{i}, \mathbf{X}^{i}\right]=0
$$

Originally [13], this quantum mechanical system was suggested as a regularized theory of a (super)membrane in flat 11-dimensional space-time in light-cone gauge. The matrices $\mathbf{X}^{i}$ play the role of embedding functions of the membrane. Regularization is removed by taking the limit $N \rightarrow \infty$. In this language $R=2 \pi l_{11}^{3}$ is the membrane tension.

Alternatively, one may consider Lagrangian (57) as an effective low-energy description for a system of $N$ D0-branes in the type IIA-theory in the $A_{0}=0$ gauge. In this case $R$ has an interpretation of compactification radius of 11-dimensional M-theory to ten dimensions and in string units, $l_{s}=1$, this radius is equal to the string coupling $g_{s}$.

It was conjectured in Ref. [14], that large- $N$ limit of the matrix model (57) describes all of the M-theory in the infinite momentum frame. Moreover, it was argued $[15,16]$ that the quantum mechanical system (57) at finite $N$ describes a sector of M-theory with $N$ units of momentum along compact light-like direction.

In Ref. [8] fuzzy cylinder was obtained as a BPS-solution in the matrix model (in $A_{0}=\mathbf{Z}$ gauge) and was interpreted as a D2-brane of type IIA theory. In Ref. [11] field configurations similar to our Eqs. (18) were discussed in this context and were interpreted as junctions of D2-branes. It is worth noting, however, that these junctions (domain walls) were not obtained as solutions of matrix model equations.

Here we would like to suggest that domain walls studied in this paper may be obtained as solutions of the matrix model in curved backgrounds.

If one sets $\mathbf{X}^{i}=0$ for $i \geq 4$ in the matrix model Lagrangian, one arrives at the action very similar to the action (12) of gauge theory on the fuzzy cylinder. The only difference is that extra terms linear in $\mathbf{X}$ and $\mathbf{Y}$ present in the definition of the field strength in this theory (see Eqs. (11b), (11c)) are absent in the matrix model. So our purpose in this section 
is to find a way to introduce these terms, as well as the term (20), into the matrix model Lagrangian.

A generalization of the matrix model Lagrangian (57) to arbitrary curved background is not known (see Ref. [17] for a discussion of this problem). However, there is a proposal [18] on how to modify the Lagrangian of the matrix model to incorporate the effect of arbitrary weakly curved background independent of the light cone coordinate $x^{-}$. Namely, to describe the effect of non-trivial eleven-dimensional metric $g_{M N}=\eta_{M N}+h_{M N}$ and three-form $A_{M N L}$ at the linear level, one adds the following terms to the Lagrangian of the matrix model

$$
\begin{aligned}
\Delta L_{g} & =\frac{1}{2} \sum \frac{1}{n !} \partial_{i_{1}} \ldots \partial_{i_{n}} h_{M N} \operatorname{STr}\left(T^{M N} \mathbf{X}^{i_{1}} \ldots \mathbf{X}^{i_{n}}\right) \\
\Delta L_{A} & =\sum \frac{1}{n !} \partial_{i_{1}} \ldots \partial_{i_{n}} A_{M N L} \operatorname{STr}\left(J^{M N L} \mathbf{X}^{i_{1}} \ldots \mathbf{X}^{i_{n}}\right)
\end{aligned}
$$

where STr stands for the totally symmetrized trace

$$
\operatorname{STr}\left(A_{a_{1}} \ldots A_{a_{n}}\right)=\frac{1}{n !} \operatorname{Tr} \sum_{\text {transmutations } \sigma} A_{\sigma\left(a_{1}\right) \ldots A_{\sigma\left(a_{n}\right)} .}
$$

We have not written terms describing magnetic interactions of the membrane and terms with fermions. The former do not appear in the backgrounds considered below, while the latter are not relevant for our purposes.

Explicit expressions for the components of the energy-momentum tensor $T^{M N}$ and antisymmetric current $J^{M N L}$ can be found in Ref. [18]. In what follows we will make use of the expressions for the $T^{++}$and $J^{+i j}$ components

$$
\begin{aligned}
T^{++} & =\frac{1}{R} \\
J^{+i j} & =-\frac{i}{6 R}\left[\mathbf{X}^{i}, \mathbf{X}^{j}\right] .
\end{aligned}
$$

Eqs. (60) and (58) suggest that a natural starting point to construct the M-theory background leading to extra terms like (20) in the matrix model action, is to consider metric with non-trivial $g_{++}$component. In order to be a legitimate background of the M-theory at least in the supergravity approximation, this metric should be supplemented with the appropriate three-form field to satisfy the equations of eleven-dimensional supergravity. Thus one naturally arrives at the following class of the supergravity solutions [19]

$$
\begin{aligned}
& d s^{2}=-2 d x^{+} d x^{-}+\sum d x^{l} d x^{l}-H\left(x^{l}\right)\left(d x^{+}\right)^{2} \\
& F_{+i j k}=\xi_{i j k}\left(x^{l}\right)
\end{aligned}
$$


where functions $H\left(x^{l}\right)$ and $\xi_{i j k}\left(x^{l}\right)$ are related as follows

$$
\partial_{i}^{2} H=\frac{1}{6} \xi_{i j k} \xi^{i j k}
$$

and

$$
\xi \equiv \frac{1}{6} \xi_{i j k} d x^{i} \wedge d x^{j} \wedge d x^{k}
$$

is closed and co-closed form. These solutions are generalizations of the homogeneous pp-wave solutions [20] which have attracted much attention recently.

To start with, let us present an example of the background admitting fuzzy cylinder as a solution of field equations. For this purpose it suffices to consider quadratic function $H\left(x^{i}\right)$. Say, one considers $H\left(x^{i}\right)$ of the following form

$$
H_{f c}\left(x^{i}\right)=l^{2}\left(\left(x^{1}\right)^{2}+\left(x^{2}\right)^{2}\right)
$$

supplemented with the following three-form

$$
\xi_{f c}=l d \omega, \quad \omega=\left(x^{1} d x^{2} \wedge d x^{3}-x^{2} d x^{1} \wedge d x^{3}\right) .
$$

Applying the rules described above, it is straightforward to check that the bosonic part of the matrix model Lagrangian in this background has the following form

$$
L_{f c}=\frac{1}{2 R} \operatorname{Tr}\left(\dot{\mathbf{X}}^{i} \dot{\mathbf{X}}^{i}+\left(\left[\mathbf{X}^{1}, \mathbf{X}^{3}\right]+i l \mathbf{X}^{2}\right)^{2}+\left(\left[\mathbf{X}^{2}, \mathbf{X}^{3}\right]-i l \mathbf{X}^{1}\right)^{2}+\left[\mathbf{X}^{1}, \mathbf{X}^{2}\right]^{2}+\ldots\right),
$$

where dots stand for non-negative terms in the potential, vanishing for zero $\mathbf{X}^{4}, \ldots, \mathbf{X}^{9}$. One immediately recognizes that the expression (63) is the same as the Lagrangian of the gauge theory on the fuzzy cylinder where $\mathbf{X}^{1}, \mathbf{X}^{2}, \mathbf{X}^{3}$ play the role of covariant coordinates $\mathbf{X}, \mathbf{Y}, \mathbf{Z}$ and $\mathbf{X}^{4}, \ldots, \mathbf{X}^{9}$ are massless adjoint scalar fields with a specific positive definite quartic potential.

To construct gravitational background leading to the matrix model admitting domain wall solutions discussed above, let us note first that for two pp-wave solutions described by functions $H_{1,2}$ and three-forms $\xi_{1,2}$, the sum $H_{1}+H_{2}$ and $\xi_{1}+\xi_{2}$ is again a solution, if there are no hyperplanes with non-vanishing fluxes for both three-forms $\xi_{1}$ and $\xi_{2}$. We have already described gravitational background leading to the gauge theory on the fuzzy cylinder, so now we have to find the function $H_{m}$ and three-form $\xi_{(m)}$ such that $\xi_{(m) 123}=0$, which give rise to extra term of the form (20) in the matrix model action.

A natural guess would be to take

$$
H_{m}=\frac{\mu^{2}}{\lambda^{2}}\left(\left(x^{1}\right)^{2}+\left(x^{2}\right)^{2}-1\right)^{2} .
$$


However, the Laplacian of this function is not positive definite,

$$
\partial_{i}^{2} H_{m}=8 \frac{\mu^{2}}{\lambda^{2}}\left(\left(x^{1}\right)^{2}+\left(x^{2}\right)^{2}-2\right)
$$

in contradiction to Eq. (61).

To get around this difficulty, one may consider background depending on larger number of coordinates. For instance, one may make the following choice of the function $H_{m}$ and three-form $\xi_{(m)}$

$$
\begin{aligned}
& H_{m}=\frac{\mu^{2}}{\lambda^{2}}\left(\left(x^{1}\right)^{2}+\left(x^{2}\right)^{2}-\left(x^{4}\right)^{2}-\left(x^{5}\right)^{2}-1\right)^{2} \\
& \xi_{(m)}=2 \sqrt{2} \frac{\mu}{\lambda} d \omega, \quad \omega=\left(x^{1} x^{4} d x^{2} \wedge d x^{5}+x^{2} x^{5} d x^{1} \wedge d x^{4}\right)
\end{aligned}
$$

The extra piece in the matrix model potential coming from this background is

$$
V_{m}=\frac{\mu^{2}}{2 \lambda^{2}} \mathrm{~S} \operatorname{Tr}\left(\left(\mathbf{X}^{1}\right)^{2}+\left(\mathbf{X}^{2}\right)^{2}-1\right)^{2}+\ldots
$$

where dots stand for the terms which are at least second order in coordinates $\mathbf{X}^{4}, \mathbf{X}^{5}$. These terms do not affect equations for configurations with $\mathbf{X}^{4}=\mathbf{X}^{5}=0$ which we are focusing on here. Now, it is straightforward to check that

$$
\frac{\mu^{2}}{2 \lambda^{2}} \mathrm{~S} \operatorname{Tr}\left(\left(\mathbf{X}^{1}\right)^{2}+\left(\mathbf{X}^{2}\right)^{2}-1\right)^{2}=\frac{\mu^{2}}{2 \lambda^{2}} \operatorname{Tr}\left(\left(\mathbf{X}^{1}\right)^{2}+\left(\mathbf{X}^{2}\right)^{2}-1\right)^{2}+\frac{\mu^{2}}{6 \lambda^{2}} \operatorname{Tr}\left[\mathbf{X}^{1}, \mathbf{X}^{2}\right]^{2}+\ldots
$$

where dots now stand for commutator terms which do not affect the field equations (but, in the $N \rightarrow \infty$ limit, in general contribute to the energy). The first term in Eq. (65) coincides with the extra term given by Eq. (20) while the second one can be eliminated by the redefinition of the parameters $\mu$ and $\lambda$ (at $\mu^{2}<\lambda^{2}$ ). Thus, Eq. (65) provides an example of the supergravity background leading to the matrix model with domain wall solutions discussed in this paper. It is clear from the discussion above that one can construct a variety of backgrounds with this property.

There is a subtlety conserning the stability of the domain wall solution in the matrix model. As we showed in section 3 , if the extra coordinates $\mathbf{X}^{4}, \ldots, \mathbf{X}^{9}$ are disregarded, the domain wall saturates the BPS bound (23), and thus it is stable. However, when the extra coordinates are included, the omitted terms in Eq. (64) may become tachyonic, in the domain wall background, along the coordinates $\mathbf{X}^{4}, \ldots, \mathbf{X}^{9}$, so that the stability may be lost. In order to ensure the stability, it is desirable to find a background admitting the domain wall solution, which saturates a BPS-type bound for the full matrix model action. 
Such background can be obtained using technique proposed in Ref. [21]. The following class of pp-wave backgrounds with four extra ${ }^{7}$ supersymmetries was described there,

$$
\begin{aligned}
& H=\left|\frac{\partial W(\phi)}{\partial \phi^{a}}\right|^{2} \\
& \xi=\frac{1}{4} d\left(\epsilon_{a b c} \frac{\partial W(\phi)}{\partial \phi^{a}} \partial \phi^{+b} \wedge \partial \phi^{+c}+\text { h.c. }\right)
\end{aligned}
$$

where $a=1,2,3$ and

$$
\begin{aligned}
& \phi^{1}=x^{1}+i x^{4} \\
& \phi^{2}=x^{2}+i x^{5} \\
& \phi^{3}=x^{6}+i x^{7} .
\end{aligned}
$$

Also, it was suggested in Ref. [21] that the matrix model potential for this background has the following form ${ }^{8}$

$$
V_{\phi}=\operatorname{Tr}\left(\frac{1}{8}\left[\Phi^{a}, \Phi^{+a}\right]^{2}+\frac{1}{2}\left|\frac{1}{2} \epsilon_{a b c}\left[\Phi^{b}, \Phi^{c}\right]+\partial_{a} \tilde{W}(\Phi)\right|^{2}\right),
$$

where $\Phi^{a}, a=1,2,3$ are matrices corresponding to the coordinates $\phi^{a}$, and superpotenial $\tilde{W}(\Phi)$ is defined as follows,

$$
\tilde{W}(\Phi)=\operatorname{STr} W(\Phi) .
$$

For quadratic superpotentials the ordering prescription following from Eqs. (67), (68) agrees with that defined by Eqs. (58), (59) $)^{9}$ For generic superpotential, the two ordering prescriptions are different. We have nothing to say about this discrepancy here. If one adopts ordering defined by Eqs. (67), (68), background siutable for our purpose is a sum (in the sense explained above) of the pp-waves described by (62) and the pp-wave given by (66) with superpotential

$$
W_{m}(\phi)=\frac{\mu}{\lambda} \phi^{3}\left(\left(\phi^{1}\right)^{2}+\left(\phi^{2}\right)^{2}-1\right) .
$$

\footnotetext{
${ }^{7}$ Generally, pp-wave background leaves unbroken 16 supersymmetries out of 32 supersymmetries present in the eleven-dimensional supergravity. These supersymmetries disappear after gauge fixing of the kappasymmetry in the supermembrane action, so the resulting matrix model is not supersymmetric. In some cases pp-wave may have extra unbroken supersymmetries, and the corresponding matrix model is expected to be supersymmetric.

${ }^{8}$ We present terms in the potential which do not contain $x^{3}, x^{8}, x^{9}$ coordinates. The latter terms are the same as in flat space.

${ }^{9}$ In particular, by choosing $W=l^{2}\left(\left(\phi^{1}\right)^{2}+\left(\phi^{2}\right)^{2}\right)$ one may obtain supersymmetric realization of the fuzzy cylinder in the matrix model (with coordinates on the fuzzy cylinder $\mathbf{X}=\operatorname{Re} \Phi_{1}, \mathbf{Y}=\operatorname{Re} \Phi_{2}, \mathbf{Z}=\operatorname{Re} \Phi_{3}$ and all other fields set equal to zero), which apparently differs from that of Ref. [8].
} 
It is straightforward to check that in this background the domain wall (26) emerges as BPS solution (with identifications $\mathbf{X}=\operatorname{Re} \Phi_{1}, \mathbf{Y}=\operatorname{Re} \Phi_{2}$ and $\mathbf{Z}=x^{3}$ and all other coordinates set equal to zero). It is worth noting, however, that $(2,2)$ supersymmetry is explicitly broken in this background by terms coming from Eqs. (62).

\section{Summary and discussion}

Let us summarize our results and discuss some open problems. In this paper we constructed and studied domain walls between vacua with different gauge groups $U\left(\mathcal{N}_{1}\right)$ and $U\left(\mathcal{N}_{2}\right)$ on one of the simplest NC manifolds, fuzzy cylinder. We demonstrated that these domain walls are characterized by a non-trivial topological charge and satisfy BPS-like equations, provided an extra term stabilizing the radius of the fuzzy cylinder is added to the action. They represent a novel class of exact NC gauge solitons in the sense that they cannot be obtained by making use of solution generation technique of Refs. [23, 24, 25, 26], and do not have commutative counterparts.

By making use of the adjoint scalar and fundamental fermion fields as probes, we studied some of the properties of the domain walls and demonstrated that these objects exhibit rich pattern of non-trivial phenomena. Namely, we addressed a question, whether fields charged under the gauge groups can penetrate from one side of the wall to the other. The result is that if $\mathcal{N}_{1}<\mathcal{N}_{2}$, fields charged under $U\left(\mathcal{N}_{1}\right)$ freely penetrate through the domain wall into the $U\left(\mathcal{N}_{2}\right)$ region where they become a part of $U\left(\mathcal{N}_{2}\right)$ multiplet. On the other hand, $U\left(\mathcal{N}_{2}\right)$ fields which are not part of the $U\left(\mathcal{N}_{1}\right)$ subalgebra experience total reflection from the domain wall. It is worth mentioning here, that the higher the mass (KK number) of the $U\left(\mathcal{N}_{2}\right)$ mode, the deeper it penetrates into the region with the $U\left(\mathcal{N}_{1}\right)$ vacuum. This effect is an illustration of the $\mathrm{UV} / \mathrm{IR}$ mixing characteristic to $\mathrm{NC}$ theories.

For fermion field we found that there is a zero mode localized on the domain wall. Wavefunction profile of this mode is a gauge invariant characteristic of the shape of the domain wall. It would be interesting to understand whether there is a $\mathrm{NC}$ analogue of the index theorem relating the existence of this zero mode to non-trivial topological properties of the domain wall.

Also we studied a non-BPS wall-antiwall configuration, dividing the cylinder into three regions with $U(1), U(2)$ and again $U(1)$ gauge theories. A somewhat unusual property of this system is the absence of the interaction potential between wall and antiwall at the classical level. Still, we found a tachyonic mode in the spectrum of perturbations about this system, 
which we expect to roll down to either $U(1)$ or $U(2)$ vacuum on the whole cylinder. The precise mechanism of the tachyon condensation deserves further study. The mass of the tachyon becomes exponentially small at large separation between the wall and antiwall.

There is a simple brane picture for the domain walls between $U\left(\mathcal{N}_{1}\right)$ and $U\left(\mathcal{N}_{2}\right)$ theories. Namely, one can think of this configuration as a stack of $\mathcal{N}_{2}-\mathcal{N}_{1}$ branes of fingerstall shape inserted into $\mathcal{N}_{1}$ cylindrical branes. Some of the properties discussed above have a natural interpretation in the D-brane language. For instance, the pattern of gauge field spectrum in the presence of the domain wall is quite transparent in the string context. Indeed, gauge fields belonging to the $U\left(\mathcal{N}_{1}\right)$ multiplet correspond to strings with both ends on the cylindrical branes; these strings do not feel the presence of the domain wall at all. Strings corresponding to other gauge fields, on the contrary, have at least one end on the fingerstall branes and are bound to them, so that they cannot propagate along the entire cylinder. On the other hand, we are not aware of the stringy interpretation of the localized fermion mode.

We suggested a way to embed the $\left(U\left(\mathcal{N}_{1}\right)-U\left(\mathcal{N}_{2}\right)\right)$ domain walls into M-theory. Namely, we suggested that they emerge as solutions of the matrix theory corresponding to the curved supergravity backgrounds which have the form of pp-waves with inhomogeneous three-form field strength. This effect can be thought of as a generalization of the Myers effect [27]. We considered two ways of introducing effects of curved backgrounds into the matrix model: one based on calculations at weak curvature, and the other relying on supersymmetry. These approaches lead to apparently different prescriptions in our case, however they both yield backgrounds admitting the domain wall solutions. The advantage of the latter approach is that it leads to the domain wall solution which saturates the BPS-type bound for the full matrix model action, and thus is stable. It is worth noting that our arguments rely on the approximation of weakly curved background; one may hope that they may apply beyond this approximation, especially taking into account that pp-wave backgrounds similar to those we discussed here were shown to be exact string backgrounds [22, 21]. We leave aside an issue of the supersymmetrization of the domain walls, though the BPS property suggests that it should be possible.

To conclude, results obtained in this paper demonstrate that the rank of the gauge group can be a non-trivial dynamical parameter in the NC gauge theories. 


\section{Acknowlegements}

We are indebted to $\mathrm{V}$. Rubakov for active collaboration at the initial stage of this work and for subsequent stimulating interest and fruitful discussions. We are grateful to D. Bak for drawing our attention to gauge theories on fuzzy cylinder. We thank G. Bonelli and A. Konechny for useful correspondence. We appreciate fruitful discussions with F. Bezrukov, D. Gorbunov, D. Levkov and G. Rubtsov. S.S. would like to thank for hospitality the DESY Theory Group, where a part of this work was done. This work has been supported in part by Russian Foundation for Basic Research, grants 02-02-17398 and Swiss Science Foundation

grant 7SUPJ062239. The work of S.D. has been supported in part by INTAS grant YS 2001-2/128. The work of S.S. has been supported in part by INTAS grant YS 2001-2/141.

\section{References}

[1] A. Konechny and A. Schwarz, Phys. Rept. 360, 353 (2002) [arXiv:hep-th/0012145]; ibid. 360, 465 (2002) [arXiv:hep-th/0107251].

[2] J. A. Harvey, "Komaba lectures on noncommutative solitons and D-branes," arXiv:hepth/0102076.

[3] M. R. Douglas and N. A. Nekrasov, Rev. Mod. Phys. 73, 977 (2001) [arXiv:hepth/0106048].

[4] I. Y. Arefeva, D. M. Belov, A. A. Giryavets, A. S. Koshelev and P. B. Medvedev, "Noncommutative field theories and (super)string field theories," arXiv:hep-th/0111208.

[5] N. Seiberg and E. Witten, JHEP 9909, 032 (1999) [arXiv:hep-th/9908142].

[6] D. J. Gross and N. A. Nekrasov, JHEP 0103, 044 (2001) [arXiv:hep-th/0010090].

[7] D. Bak, K. M. Lee and J. H. Park, Phys. Lett. B 501, 305 (2001) [arXiv:hep-th/0011244].

[8] D. Bak and K. M. Lee, Phys. Lett. B 509, 168 (2001) [arXiv:hep-th/0103148].

[9] S. V. Demidov, S. L. Dubovsky, V. A. Rubakov and S. M. Sibiryakov, "Gauge theory solitons on noncommutative cylinder," arXiv:hep-th/0301047. 
[10] M. Chaichian, A. Demichev, P. Presnajder and A. Tureanu, Eur. Phys. J. C 20, 767 (2001) [arXiv:hep-th/0007156].

M. Chaichian, A. Demichev, P. Presnajder, M. M. Sheikh-Jabbari and A. Tureanu, Nucl. Phys. B 611, 383 (2001) [arXiv:hep-th/0101209].

[11] D. Bak and S. W. Kim, Nucl. Phys. B 622, 95 (2002) [arXiv:hep-th/0108207].

[12] W. I. Taylor, "The M(atrix) model of M-theory," arXiv:hep-th/0002016.

[13] B. de Wit, J. Hoppe and H. Nicolai, Nucl. Phys. B 305, 545 (1988).

[14] T. Banks, W. Fischler, S. H. Shenker and L. Susskind, Phys. Rev. D 55, 5112 (1997) [arXiv:hep-th/9610043].

[15] A. Sen, Adv. Theor. Math. Phys. 2, 51 (1998) [arXiv:hep-th/9709220].

[16] N. Seiberg, Phys. Rev. Lett. 79, 3577 (1997) [arXiv:hep-th/9710009].

[17] M. R. Douglas, Nucl. Phys. Proc. Suppl. 68, 381 (1998) [arXiv:hep-th/9707228].

[18] W. I. Taylor and M. Van Raamsdonk, Nucl. Phys. B 558, 63 (1999) [arXiv:hepth/9904095].

[19] C. M. Hull, Phys. Lett. B 139, 39 (1984).

[20] D. Berenstein, J. M. Maldacena and H. Nastase, JHEP 0204, 013 (2002) [arXiv:hepth/0202021].

[21] G. Bonelli, Nucl. Phys. B 649, 130 (2003) [arXiv:hep-th/0209225].

[22] J. G. Russo and A. A. Tseytlin, JHEP 0209, 035 (2002) [arXiv:hep-th/0208114].

[23] A. P. Polychronakos, Phys. Lett. B 495, 407 (2000) [arXiv:hep-th/0007043].

[24] D. Bak, Phys. Lett. B 495, 251 (2000) [arXiv:hep-th/0008204].

[25] M. Aganagic, R. Gopakumar, S. Minwalla and A. Strominger, JHEP 0104, 001 (2001) [arXiv:hep-th/0009142].

[26] J. A. Harvey, P. Kraus and F. Larsen, JHEP 0012, 024 (2000) [arXiv:hep-th/0010060].

[27] R. C. Myers, JHEP 9912, 022 (1999) [arXiv:hep-th/9910053]. 